\title{
Introduction to Likelihood
}

Meaningful Modeling of Epidemiologic Data, 2011

AIMS, Muizenberg, South Africa

Steve Bellan

MPH Epidemiology

PhD Candidate

Department of Environmental Science, Policy \& Management

University of California at Berkeley 
In a population of $1,000,000$ people with a true prevalence of $30 \%$, the probability distribution of number of positive individuals if 100 are sampled:

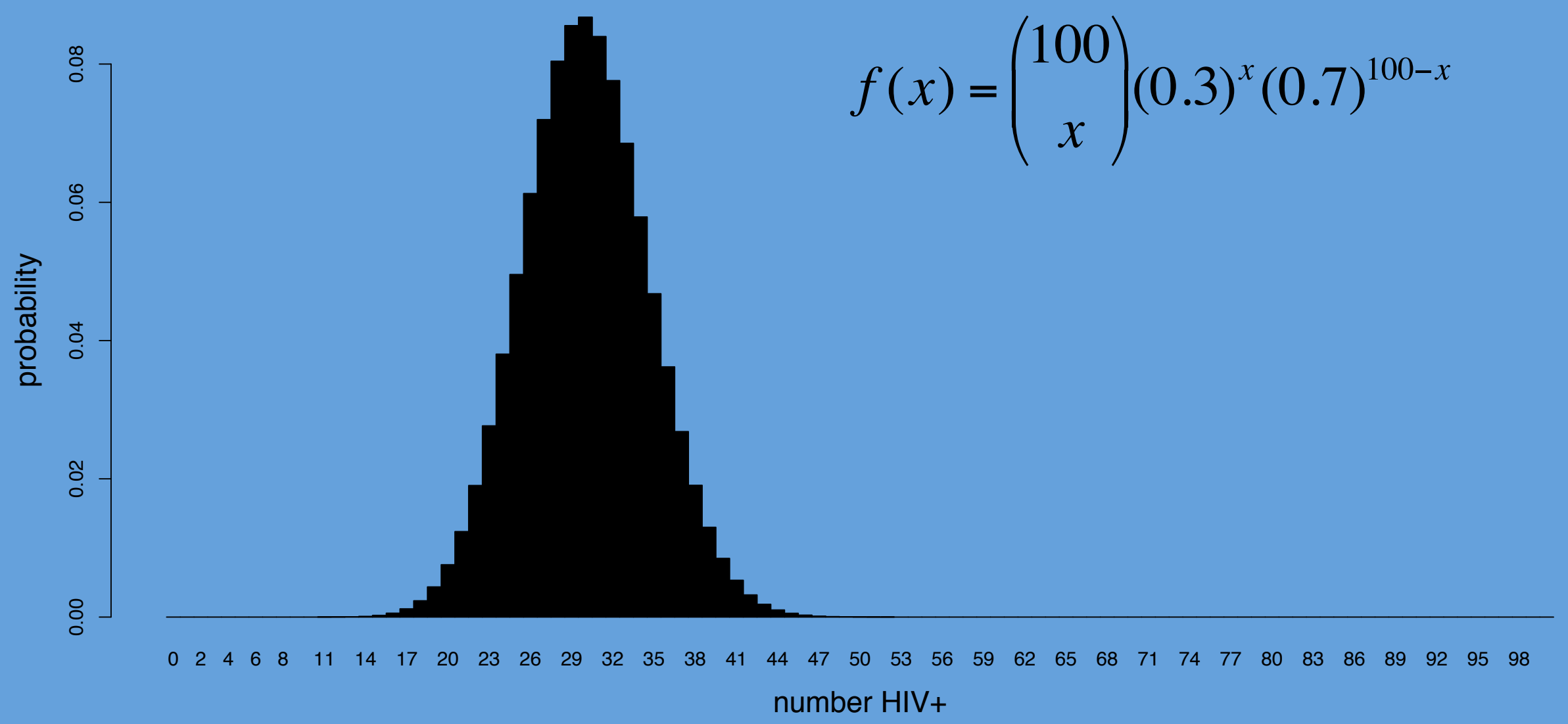

barplot $(\mathrm{dbinom}(\mathrm{x}=0: 100$, size $=100$, prob $=.3)$, names.arg $=0$ :size $)$ 
In a population of $1,000,000$ people with a true prevalence of $30 \%$, the probability distribution of number of positive individuals if 100 are sampled:

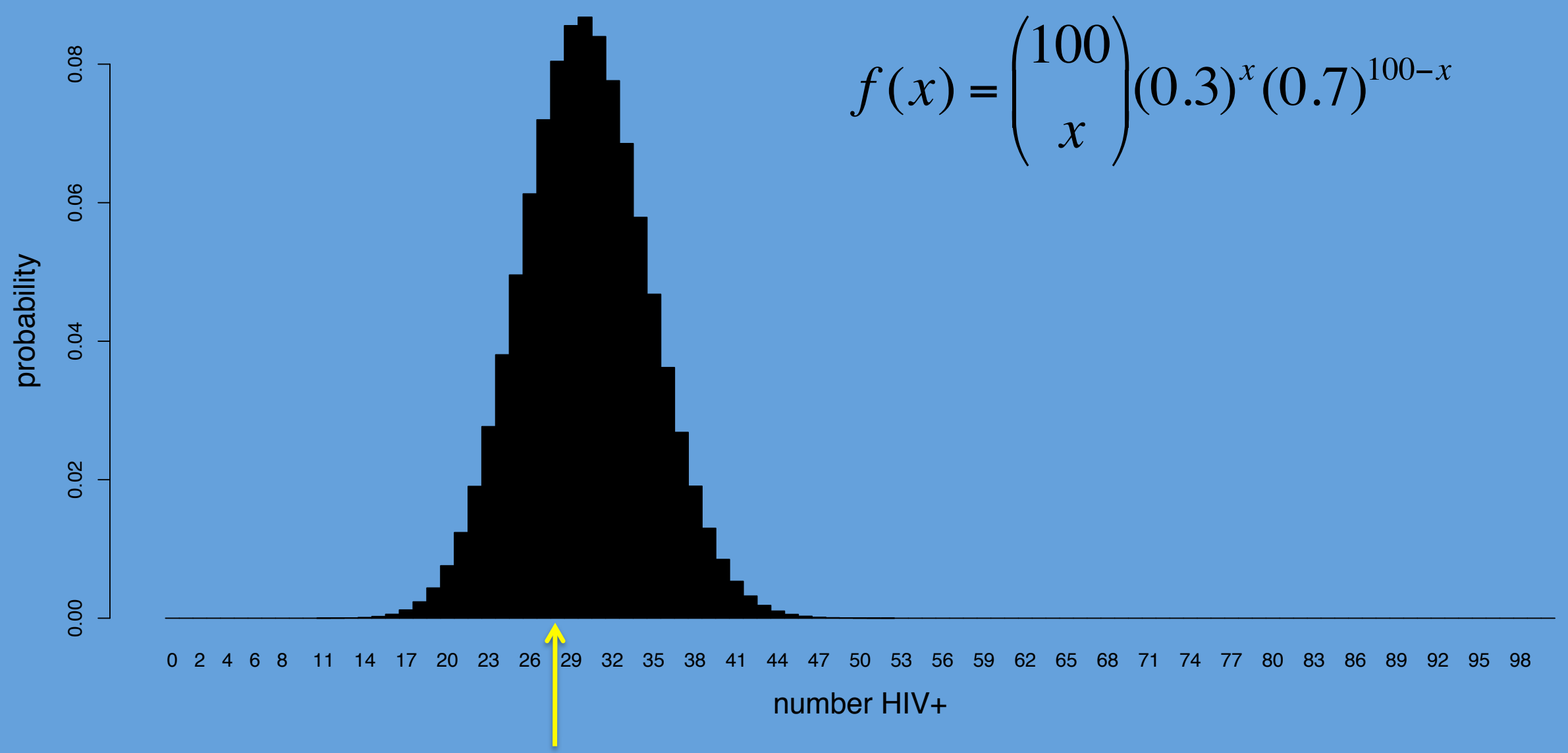

We sample 100 people once and 28 are positive:

$>\operatorname{rbinom}(\mathrm{n}=1$, size $=100$, prob $=.3)$

[1] 28 


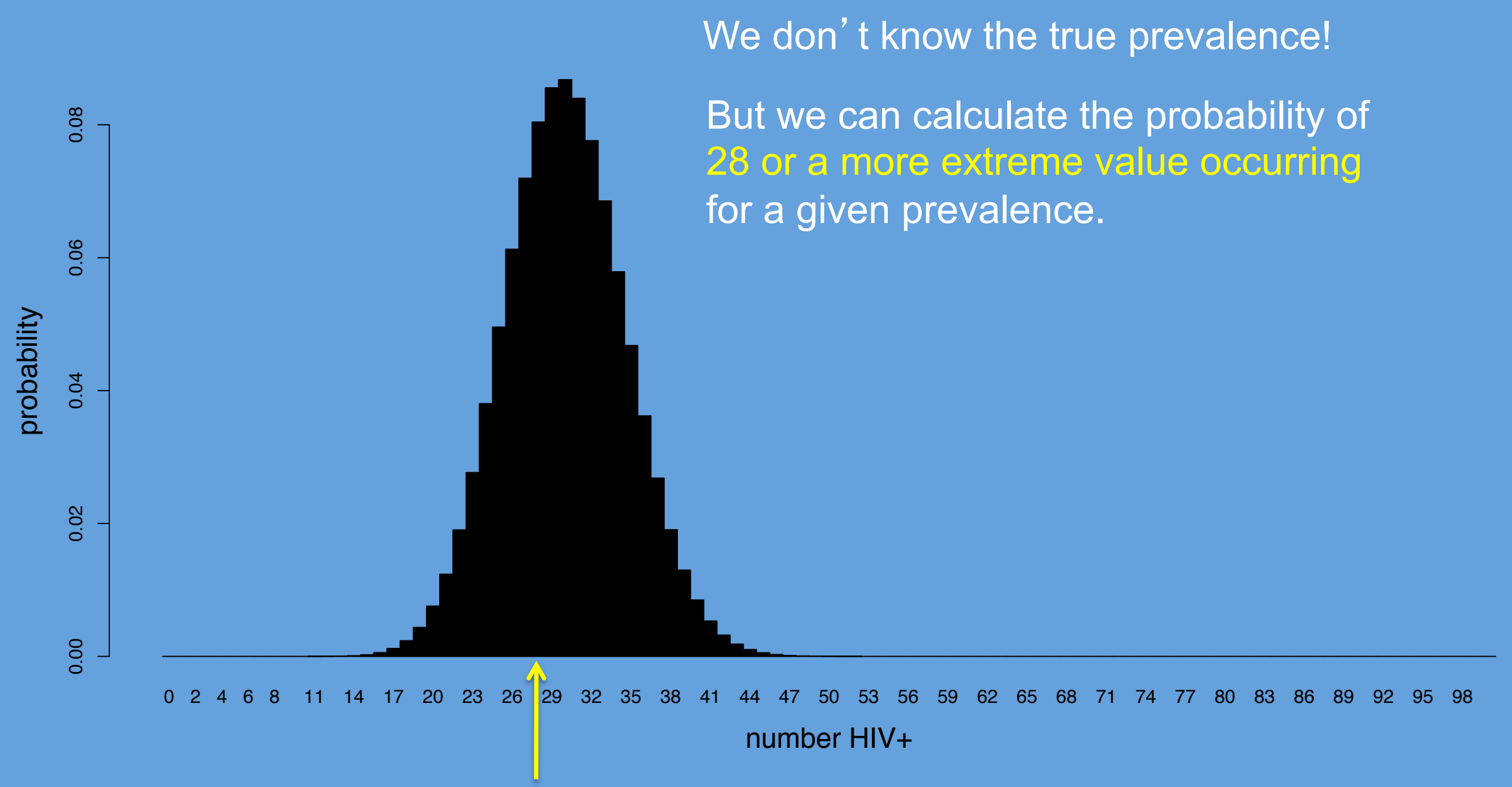

We sample 100 people once and 28 are positive:

$>\operatorname{rbinom}(\mathrm{n}=1$, size $=100$, prob $=.3)$

[1] 28 


\section{Cumulative Probability \& P Values}

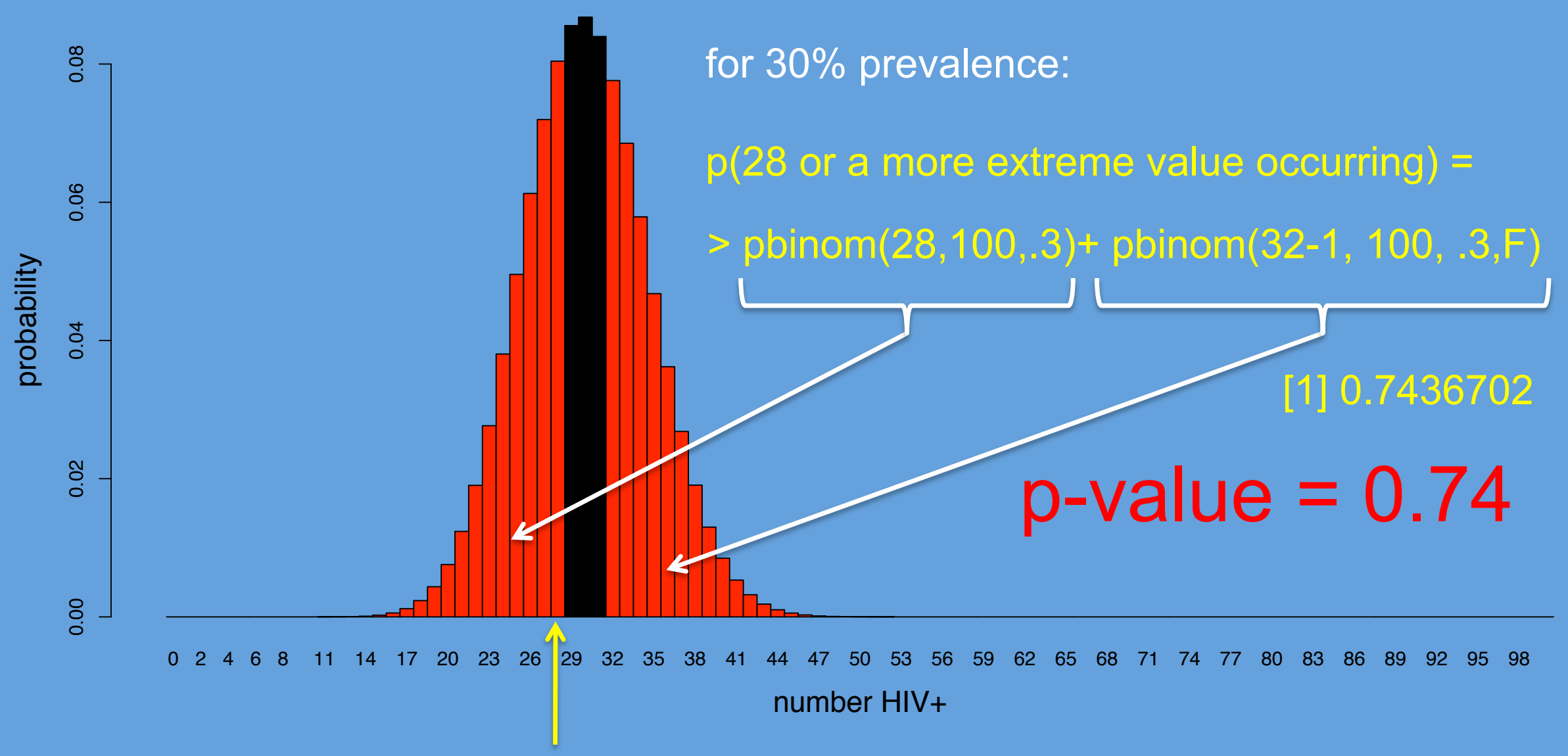

We sample 100 people once and 28 are positive. 
If true prevalence were $15 \%$, then $\mathbf{p}(28$ or more extreme) is

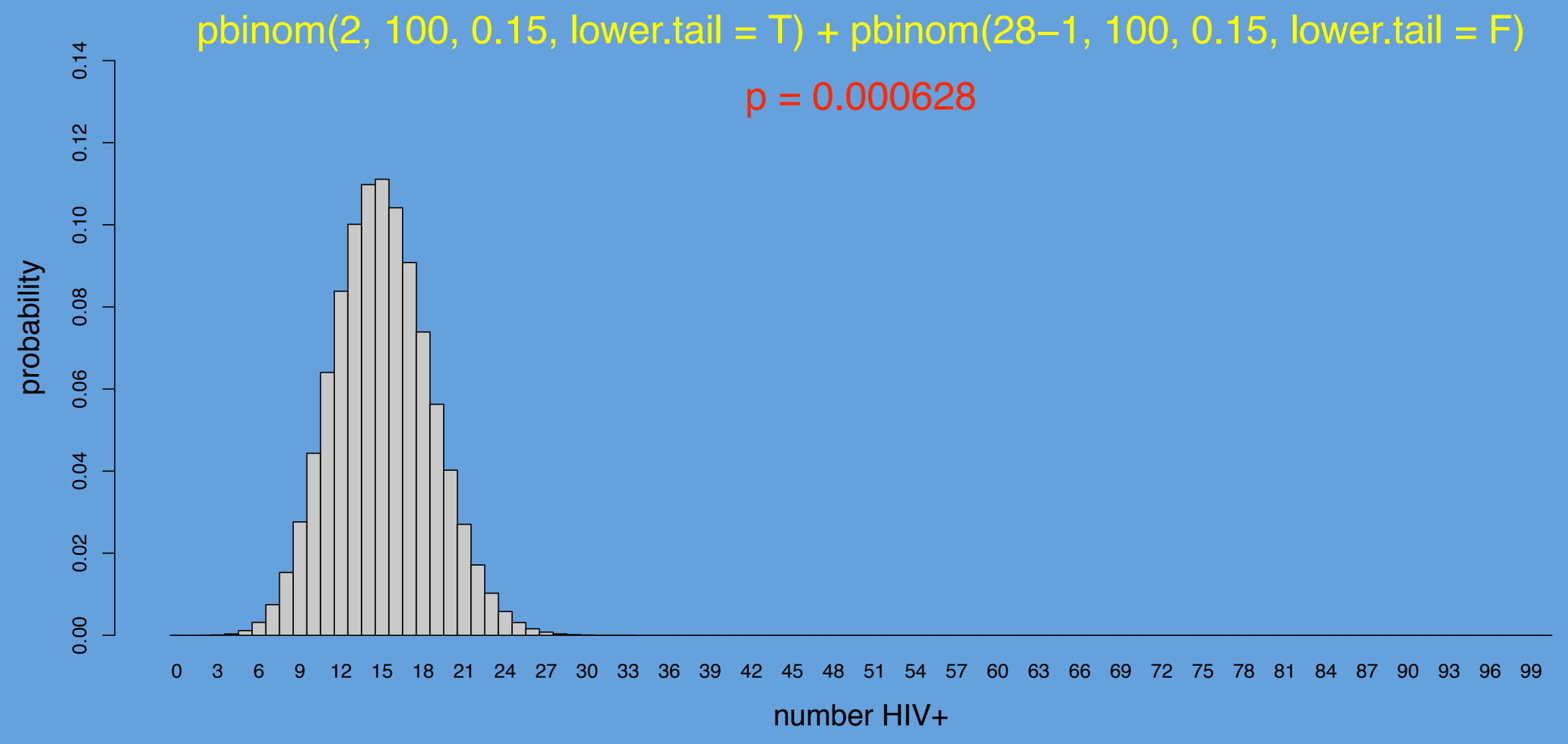


If true prevalence were $\mathbf{2 0} \%$, then $\mathbf{p}$ (28 or more extreme) is

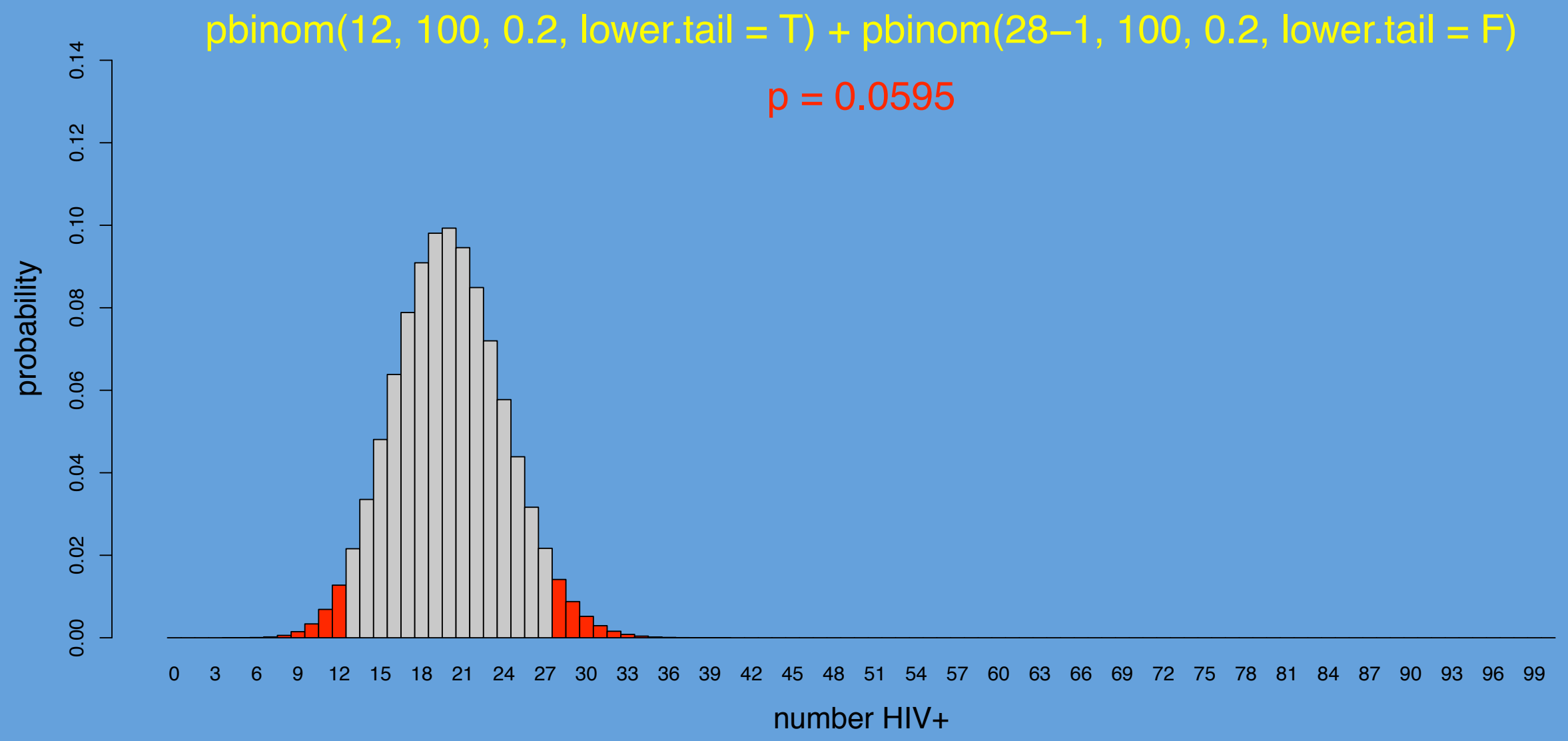


If true prevalence were $\mathbf{2 5 \%}$, then $\mathbf{p}$ (28 or more extreme) is

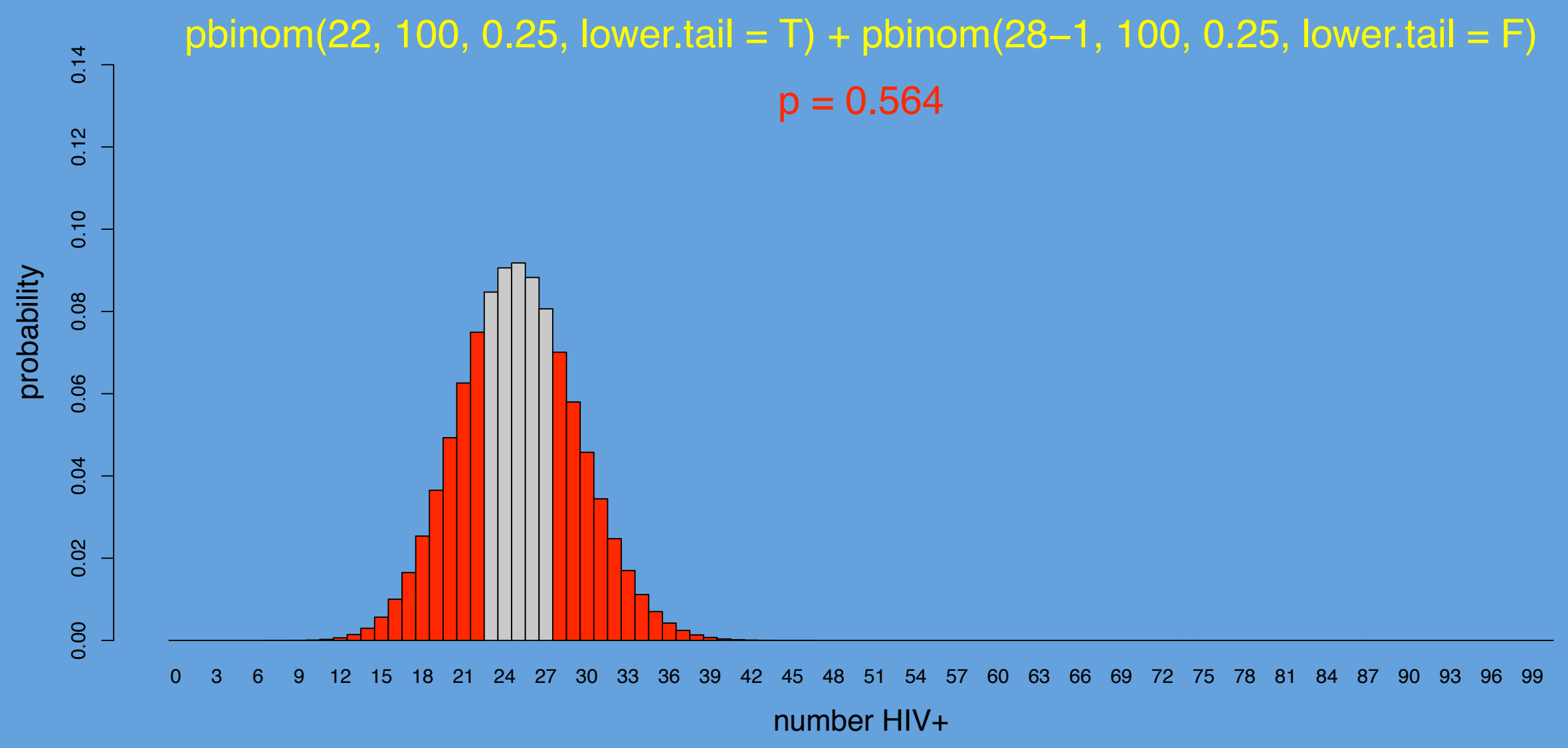


If true prevalence were $\mathbf{3 0} \%$, then $\mathbf{p}(\mathbf{2 8}$ or more extreme) is

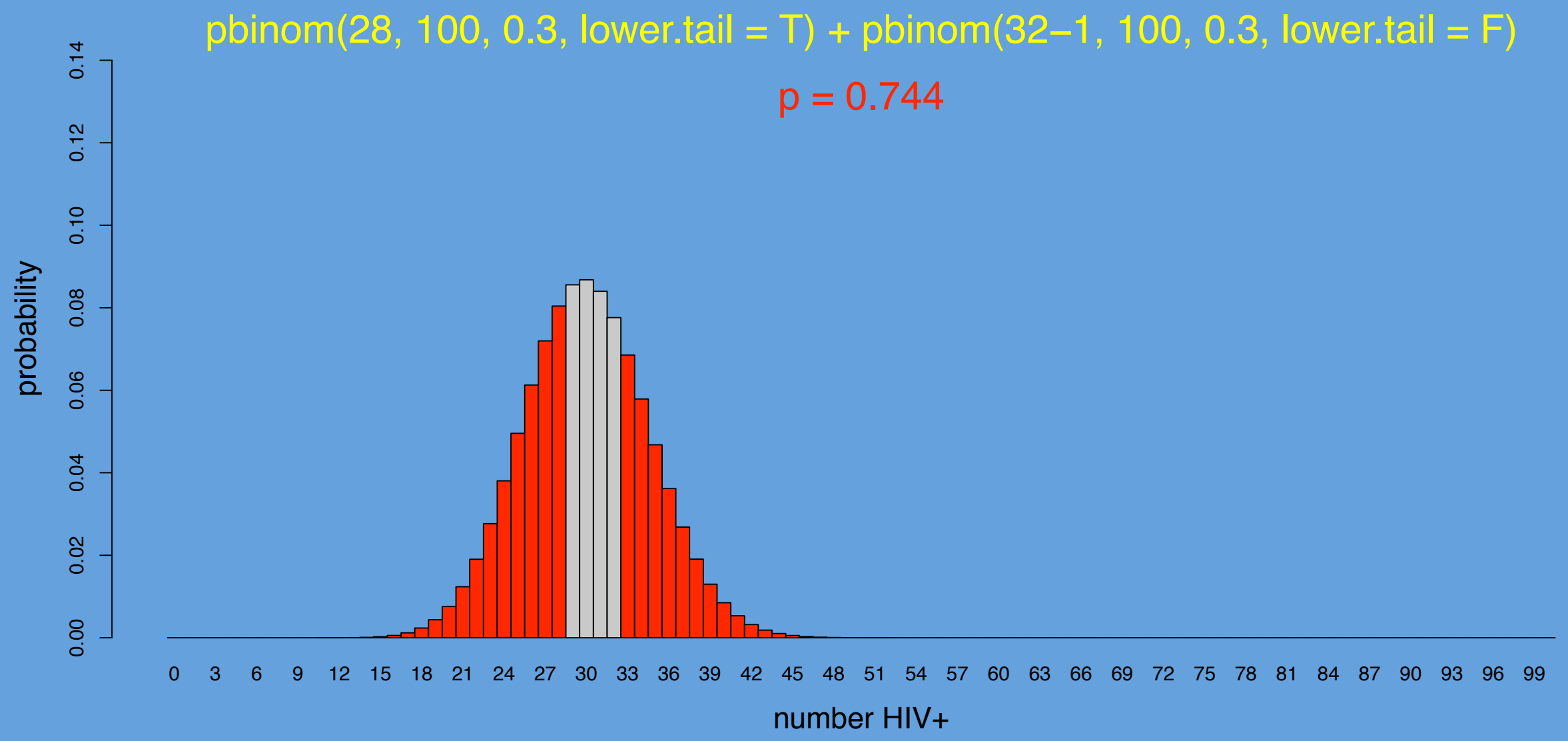


If true prevalence were $35 \%$, then $\mathbf{p}(\mathbf{2 8}$ or more extreme) is

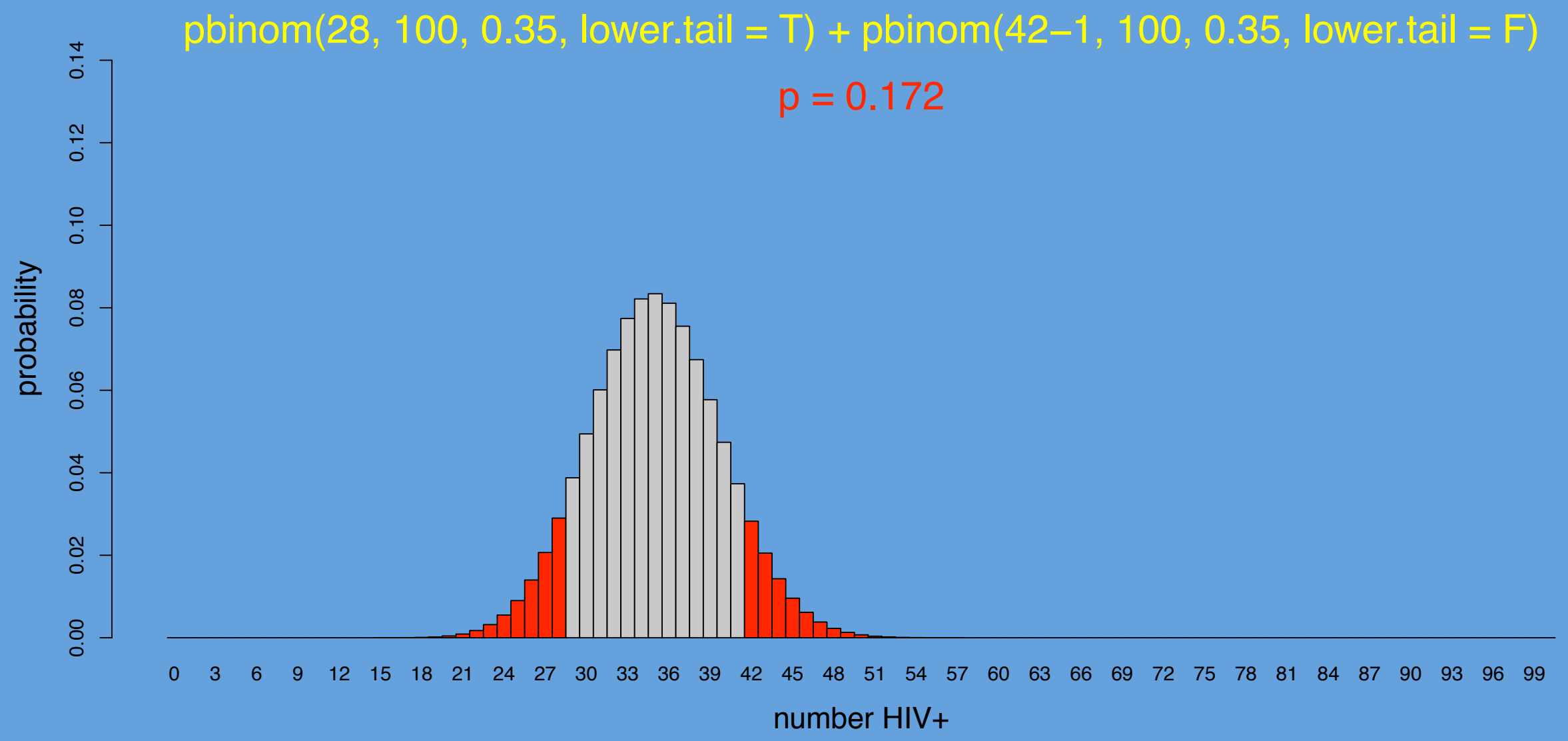


If true prevalence were $\mathbf{4 0} \%$, then $\mathbf{p}(\mathbf{2 8}$ or more extreme) is

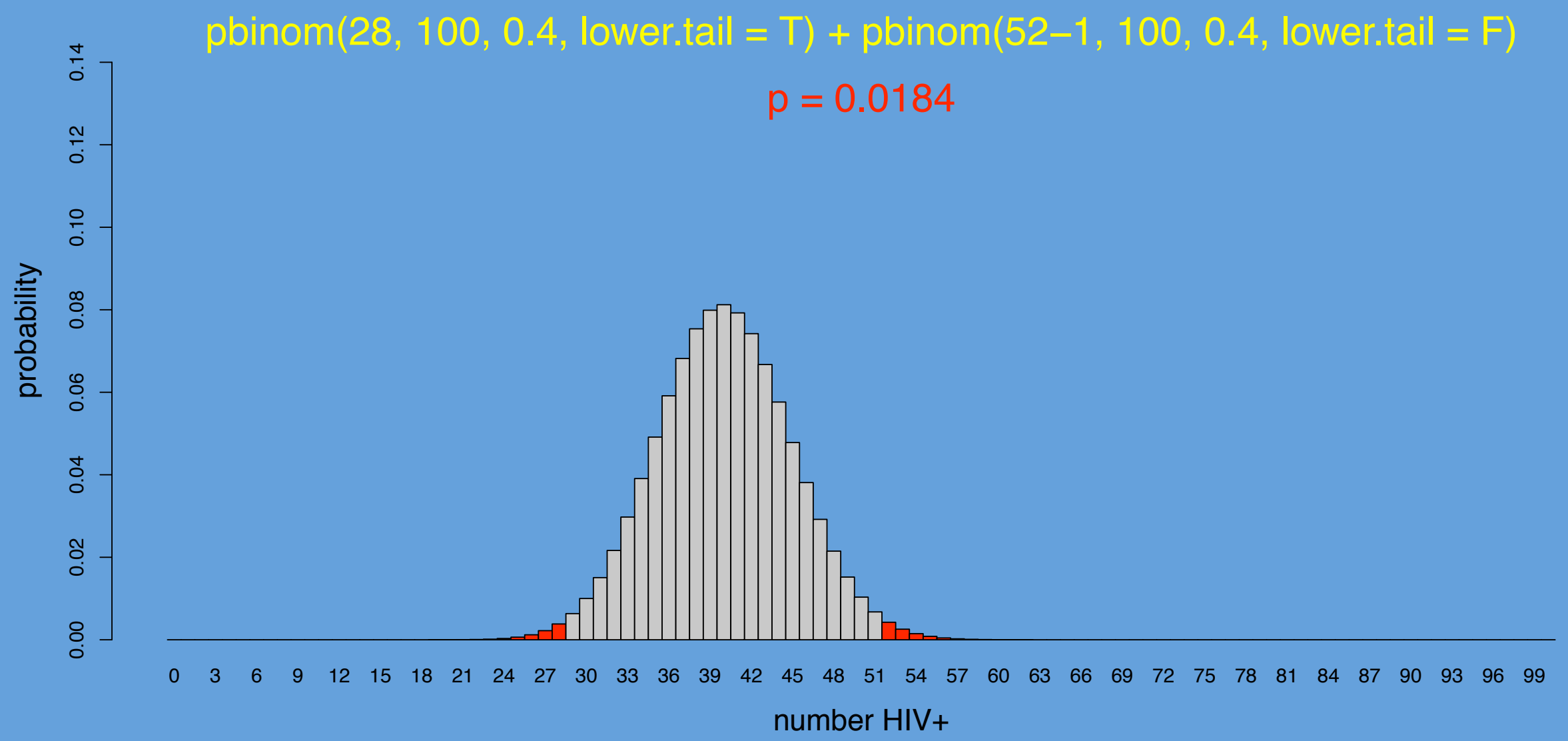


hypothetical prevalence: $15 \%$

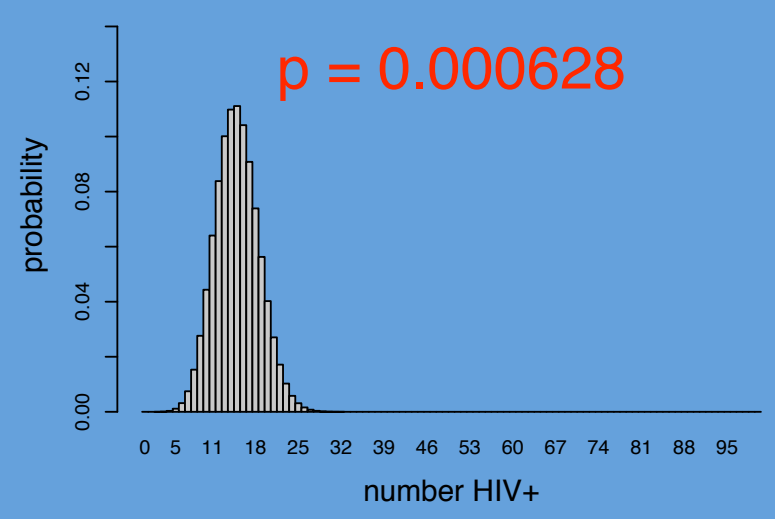

hypothetical prevalence: $30 \%$

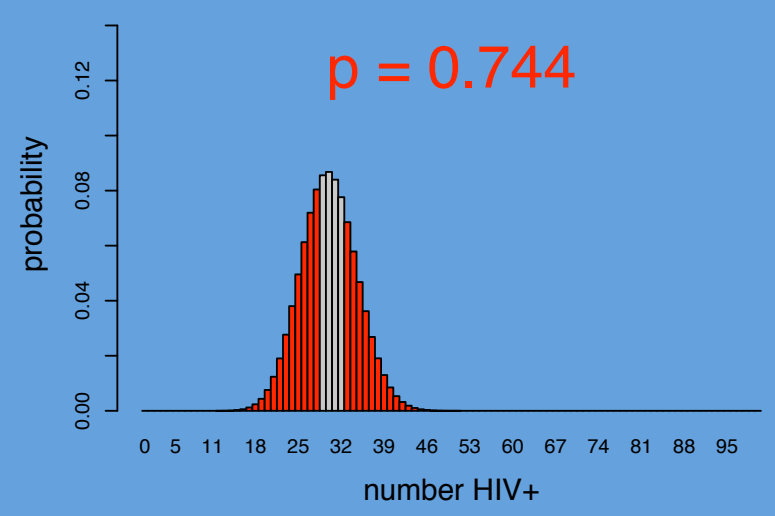

hypothetical prevalence: $20 \%$

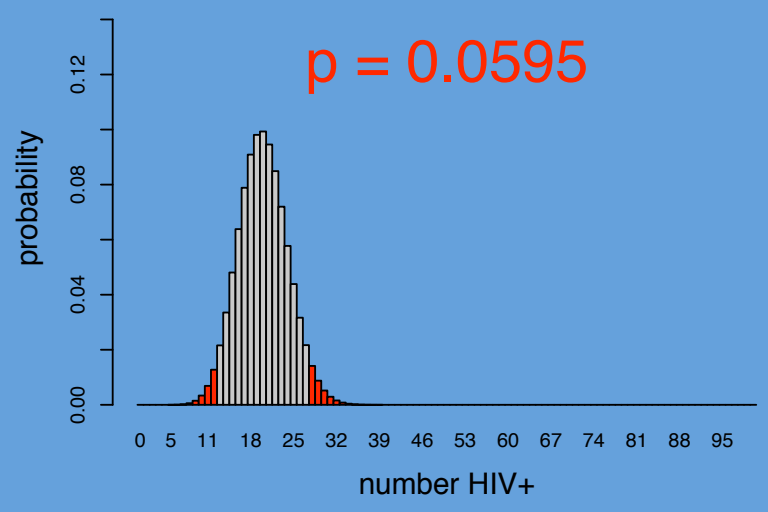

hypothetical prevalence: $35 \%$

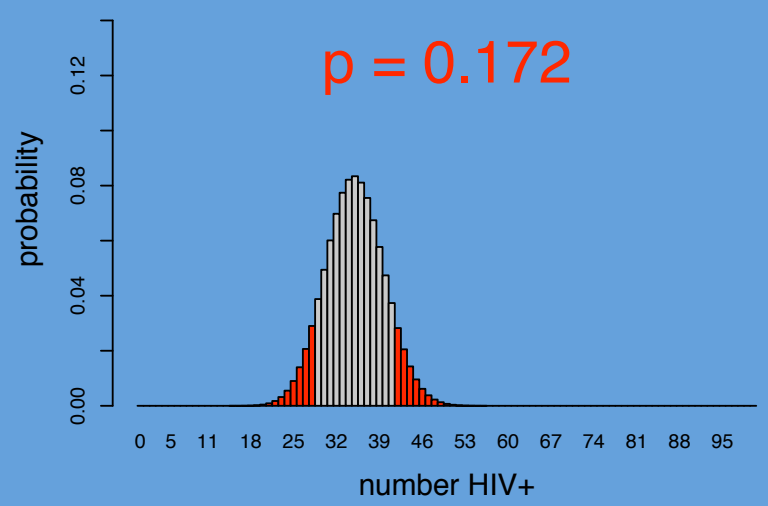

hypothetical prevalence: $25 \%$

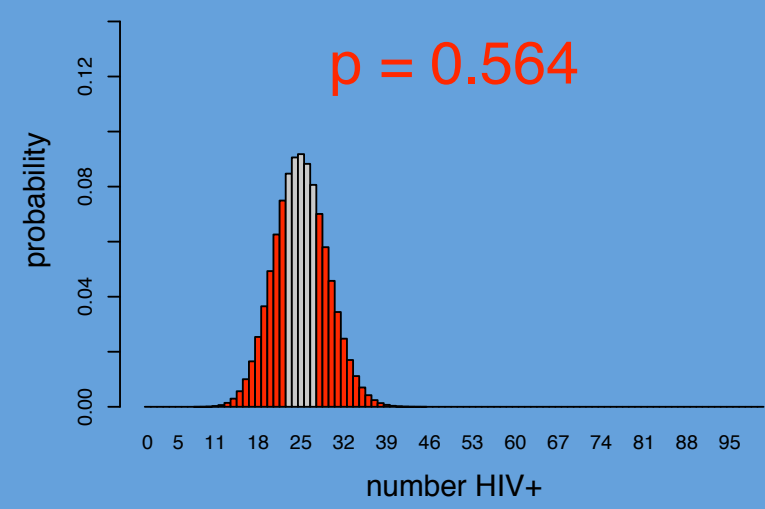

hypothetical prevalence: $40 \%$

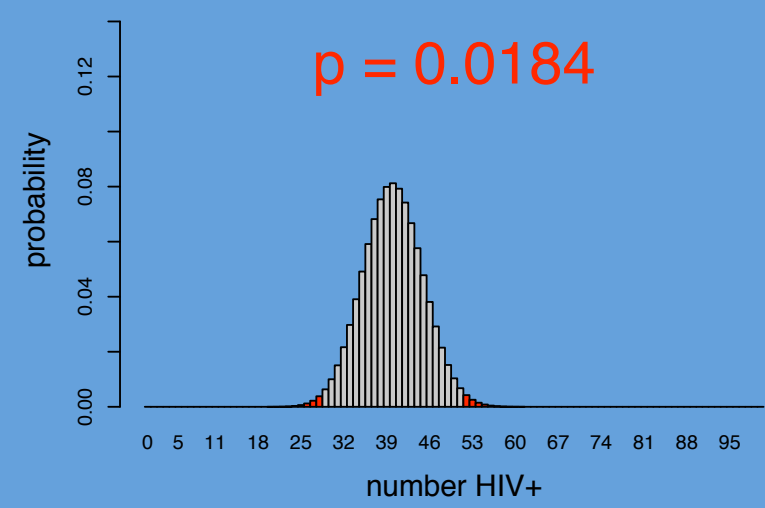




\title{
Which hypotheses do we reject?
}

IF GIVEN THE HYPOTHESIS

\author{
$p$ value $<$ cutoff
}

THEN REJECT HYPOTHESIS

Cutoff usually chosen as $\alpha=0.05$ 


\section{Which hypotheses do we reject?}

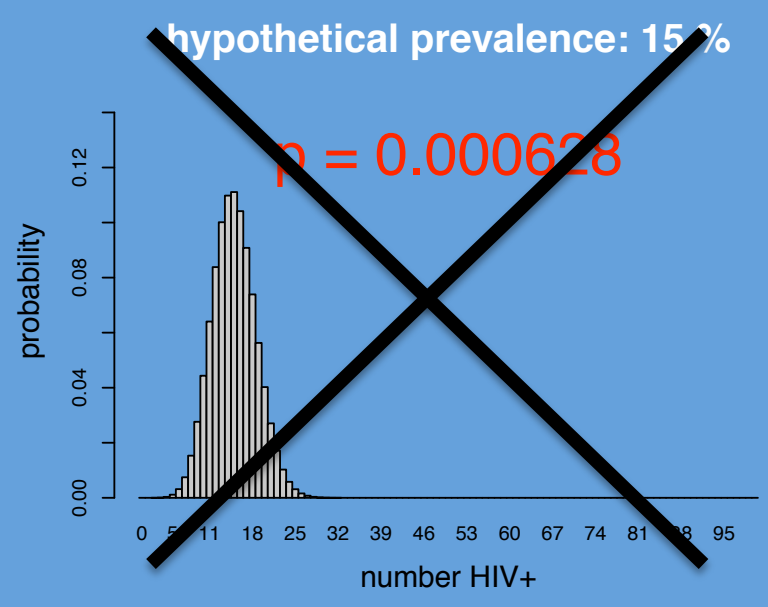

hypothetical prevalence: $30 \%$

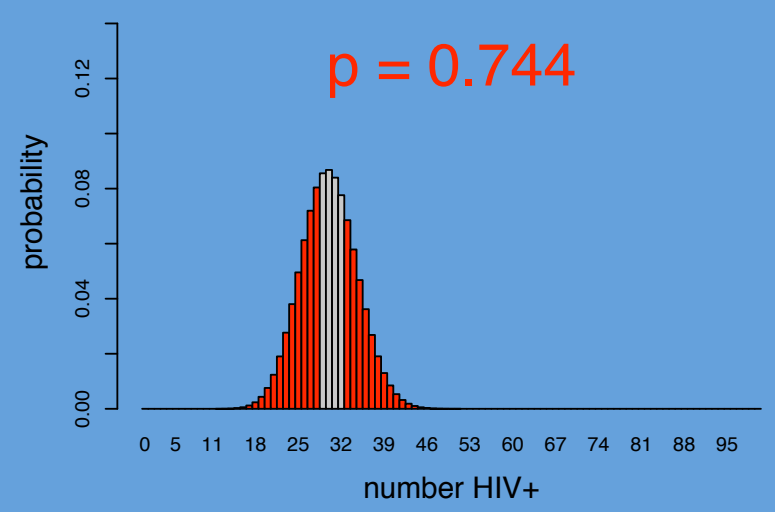

hypothetical prevalence: $20 \%$

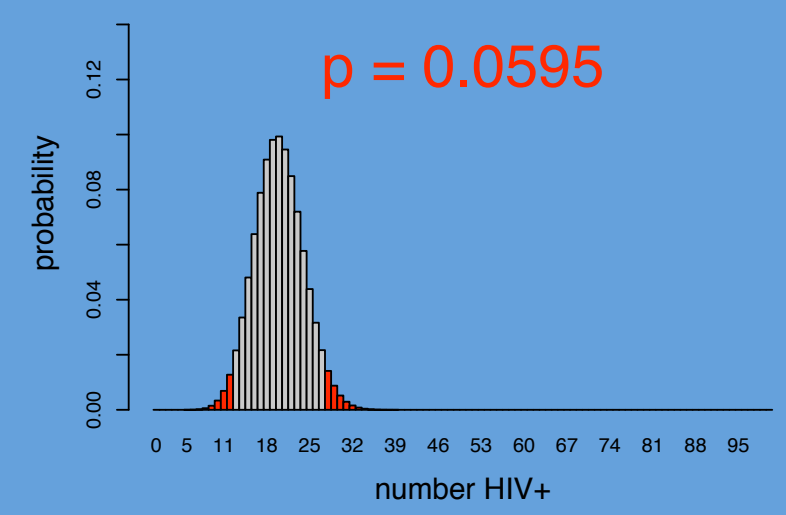

hypothetical prevalence: $35 \%$

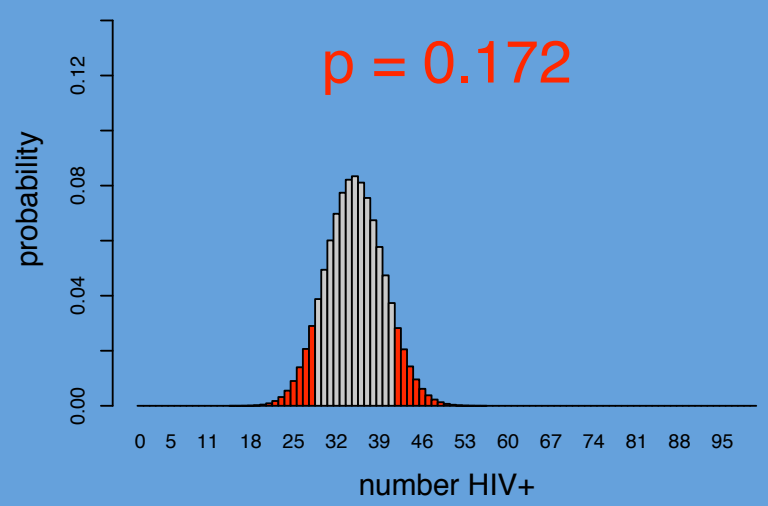

hypothetical prevalence: $25 \%$
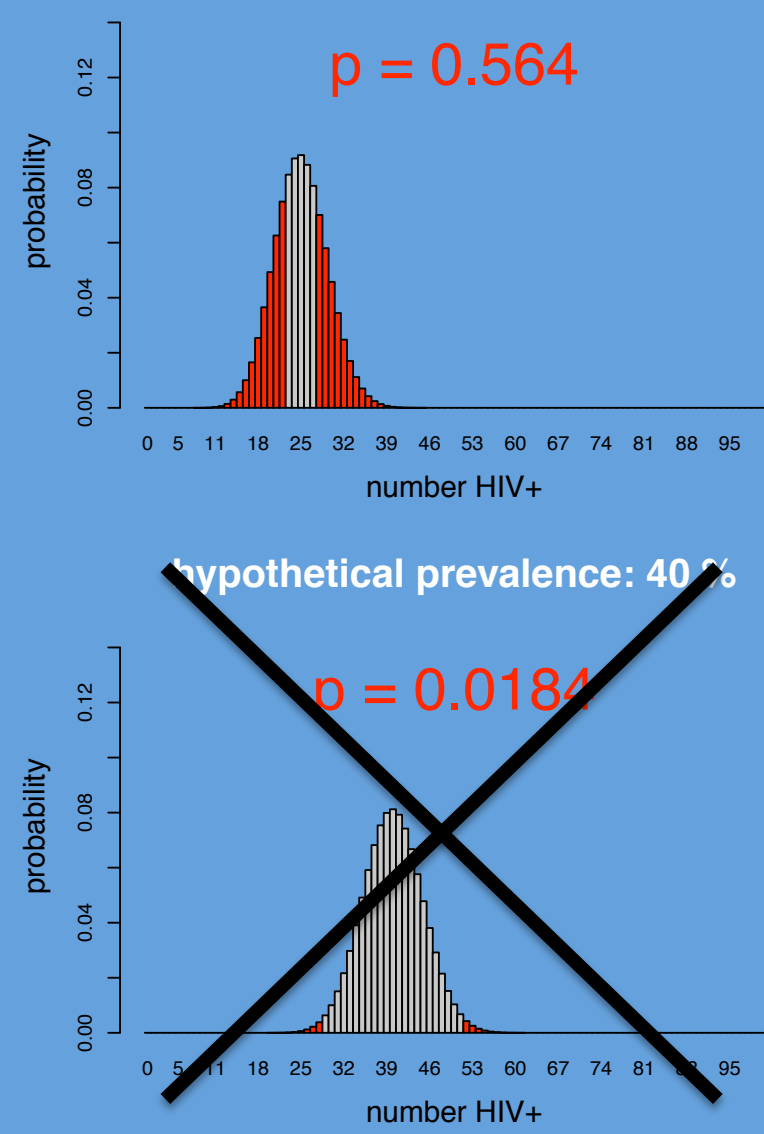


\section{Which hypotheses do we NOT reject: CONFIDENCE INTERVAL}

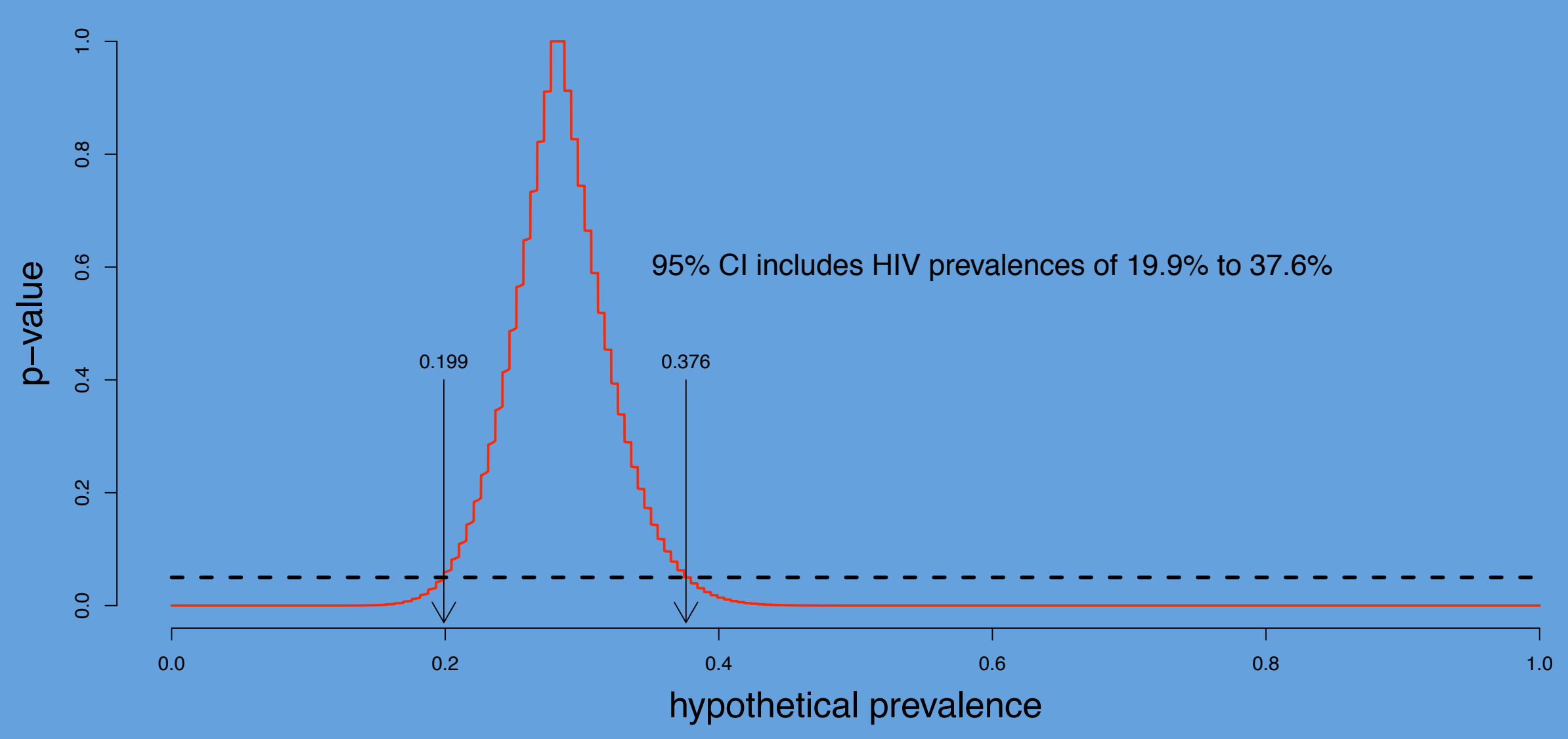




\section{Let's take another approach}

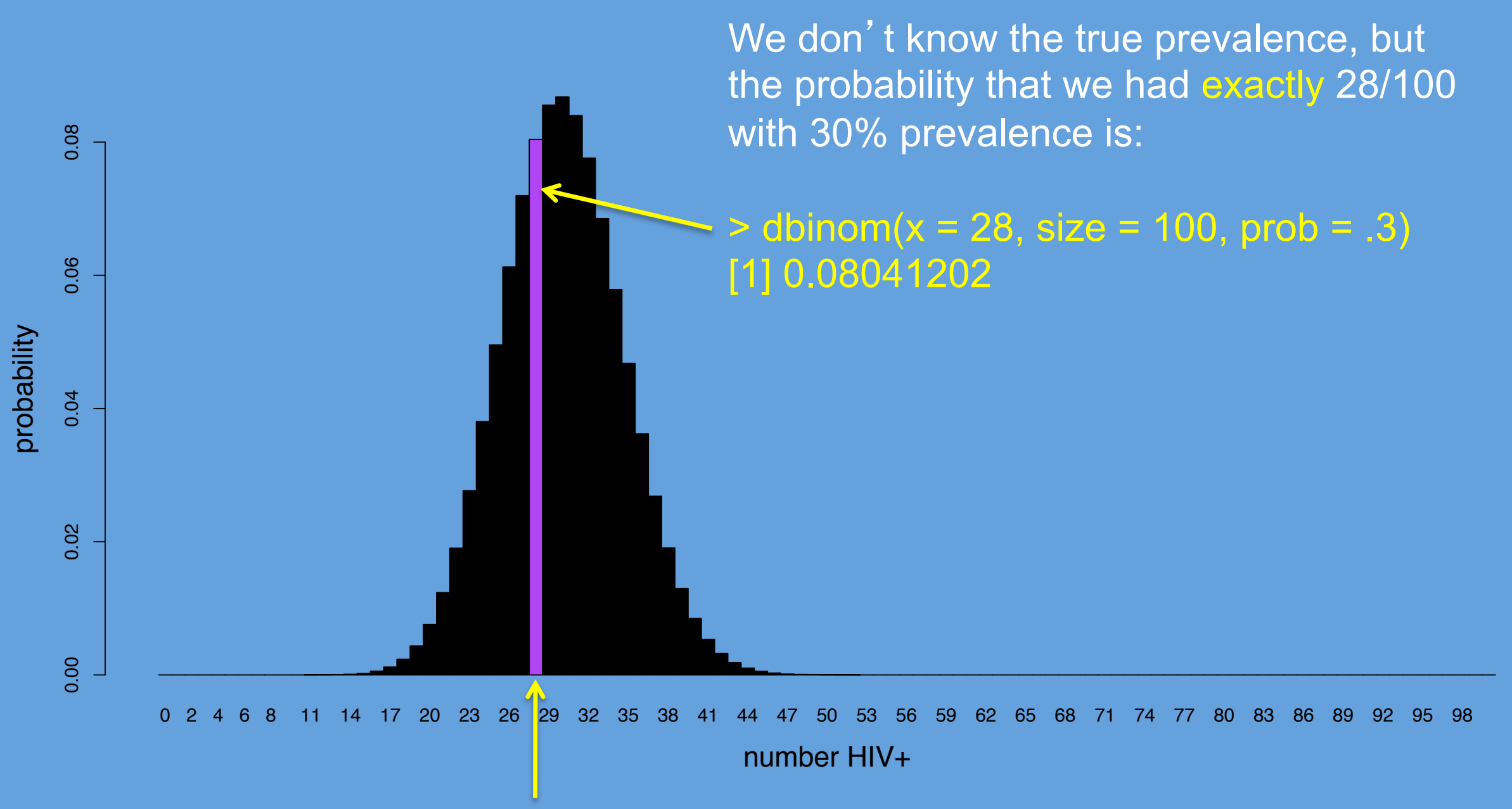

We sample 100 people once and 28 are positive:

$>\operatorname{rbinom}(\mathrm{n}=1$, size $=100$, prob $=.3)$

[1] 28 
hypothetical prevalence: $15 \%$

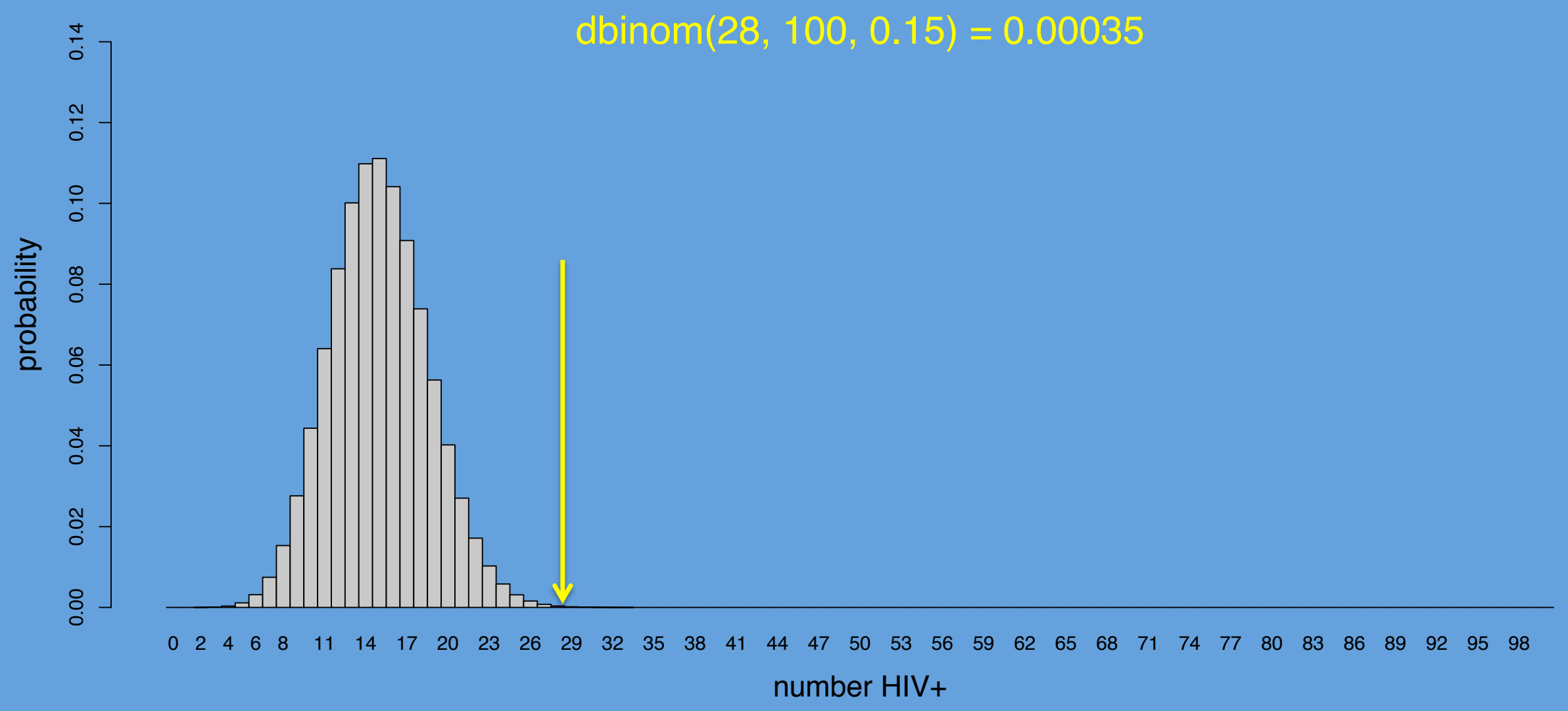


hypothetical prevalence: $\mathbf{2 0} \%$

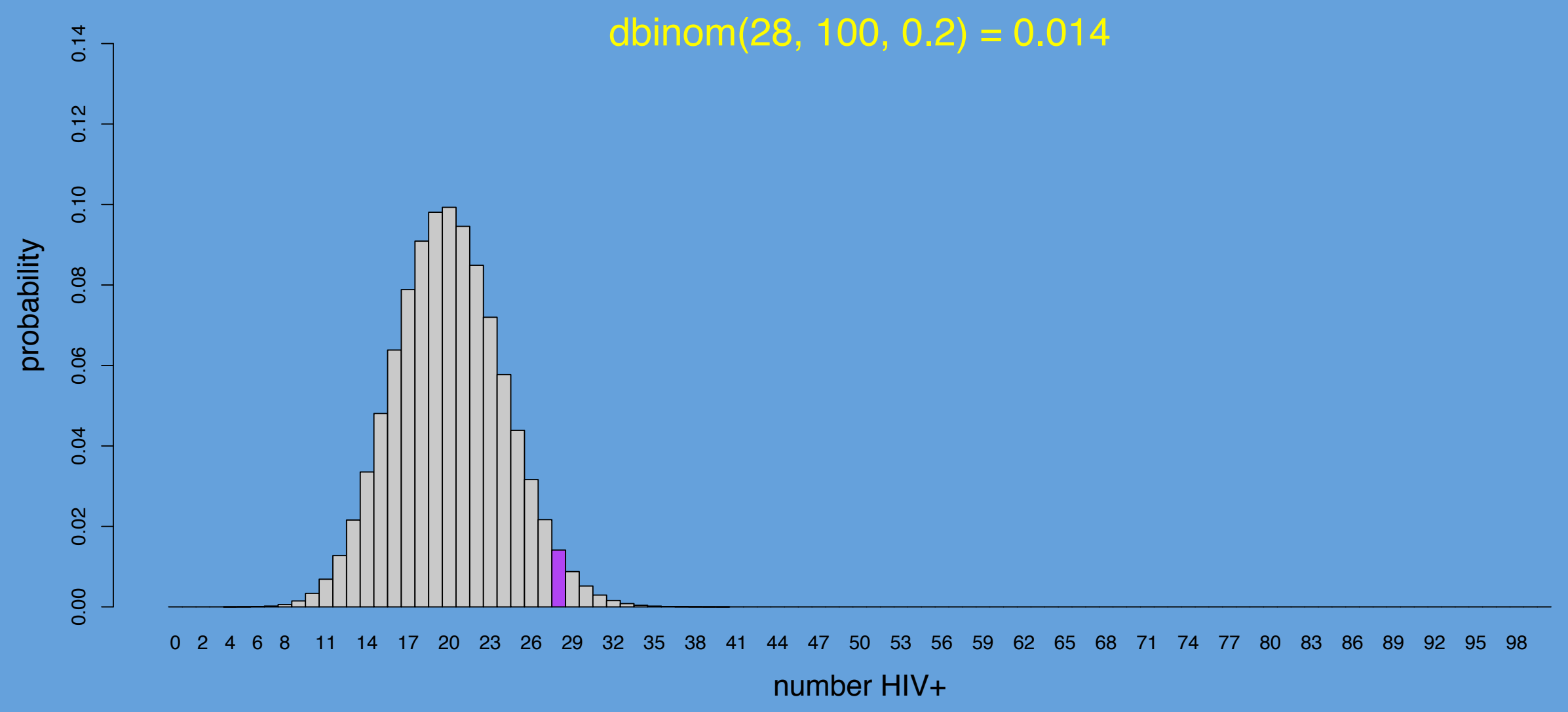


hypothetical prevalence: $\mathbf{2 5} \%$

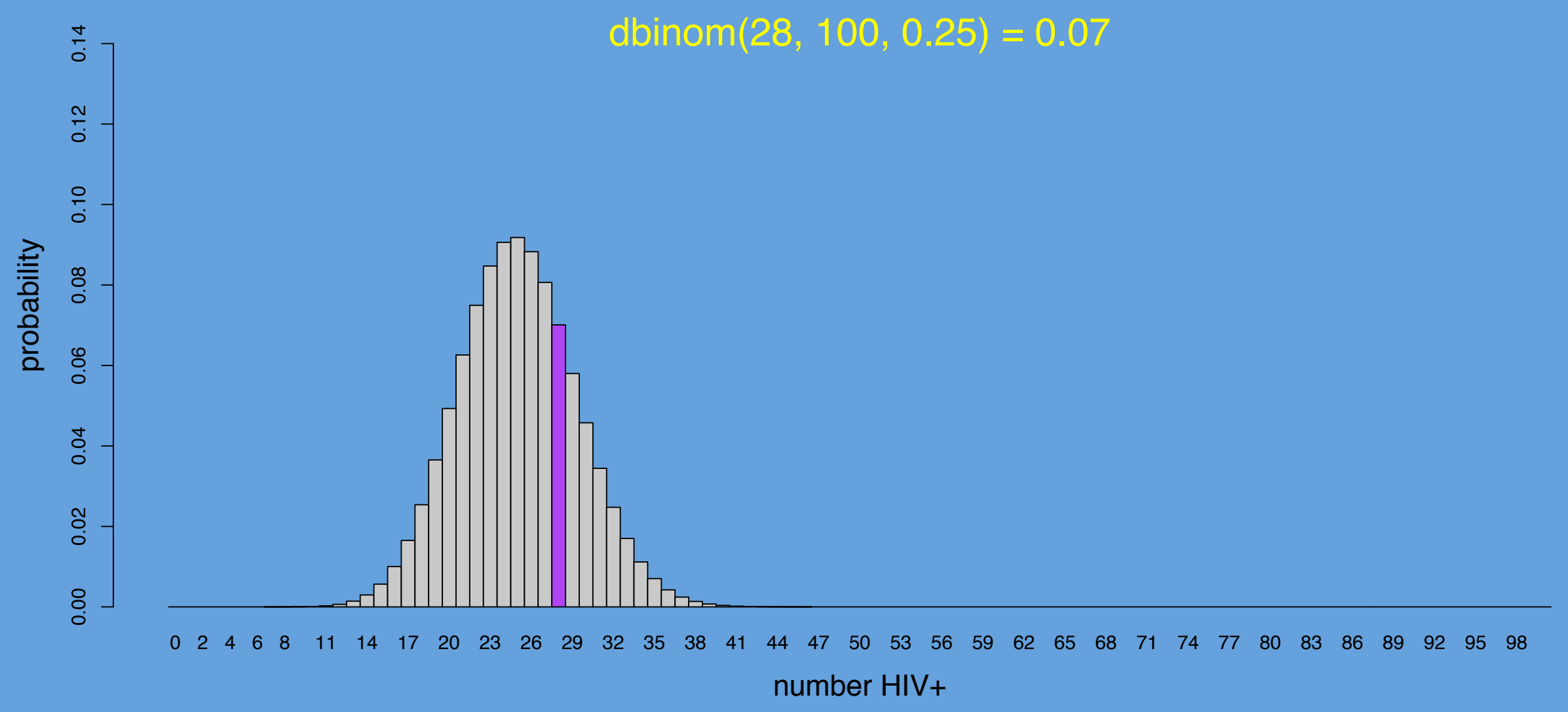




\section{hypothetical prevalence: $30 \%$}

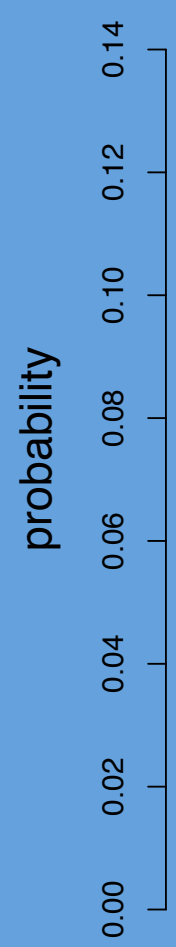

dbinom $(28,100,0.3)=0.08$

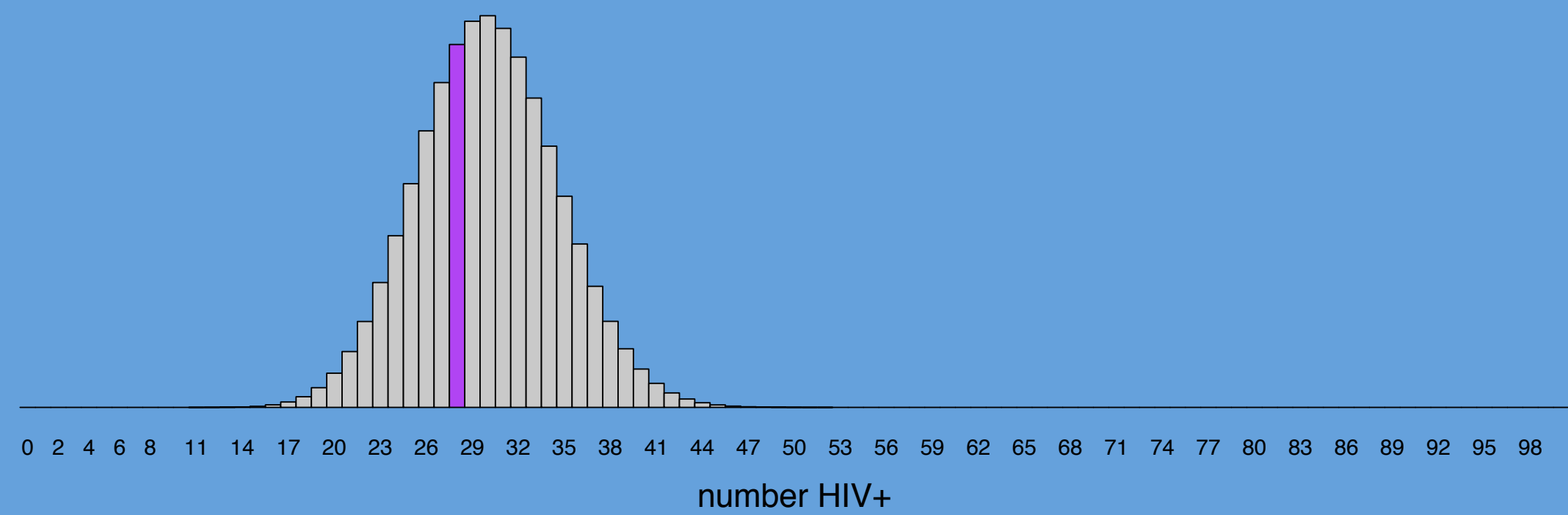




\section{hypothetical prevalence: $35 \%$}

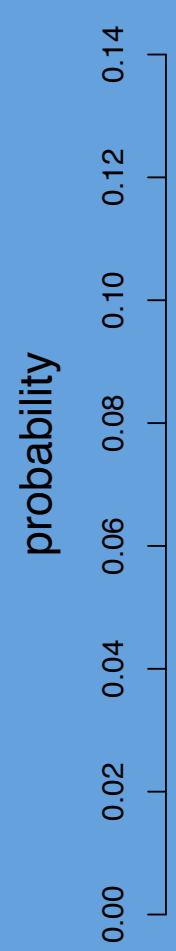

dbinom $(28,100,0.35)=0.029$

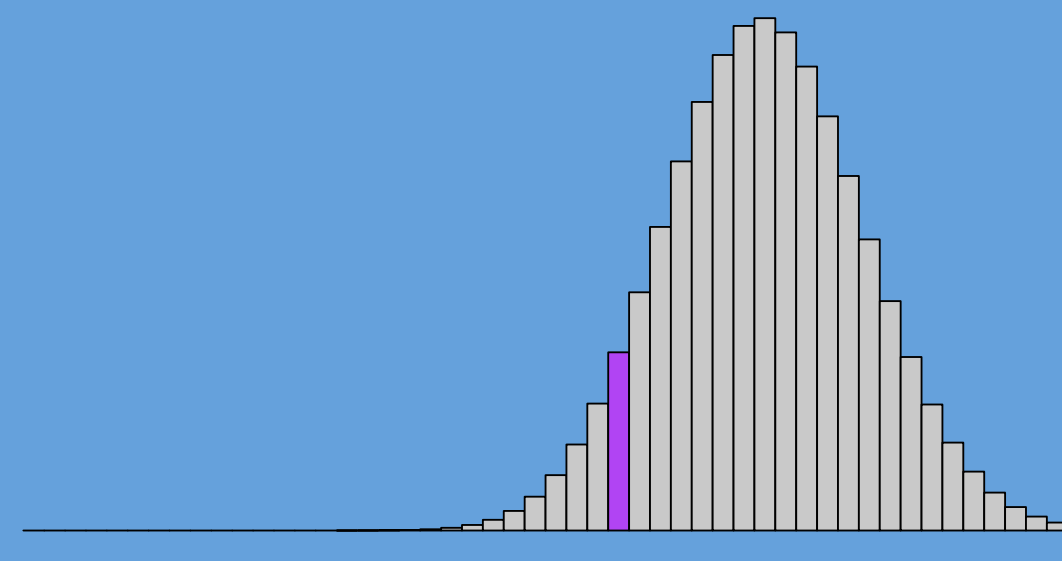

$\begin{array}{lllllllllllllllllllllllllllllllllll}0 & 2 & 4 & 6 & 8 & 11 & 14 & 17 & 20 & 23 & 26 & 29 & 32 & 35 & 38 & 41 & 44 & 47 & 50 & 53 & 56 & 59 & 62 & 65 & 68 & 71 & 74 & 77 & 80 & 83 & 86 & 89 & 92 & 95 & 98\end{array}$ number HIV+ 


\section{hypothetical prevalence: $40 \%$}

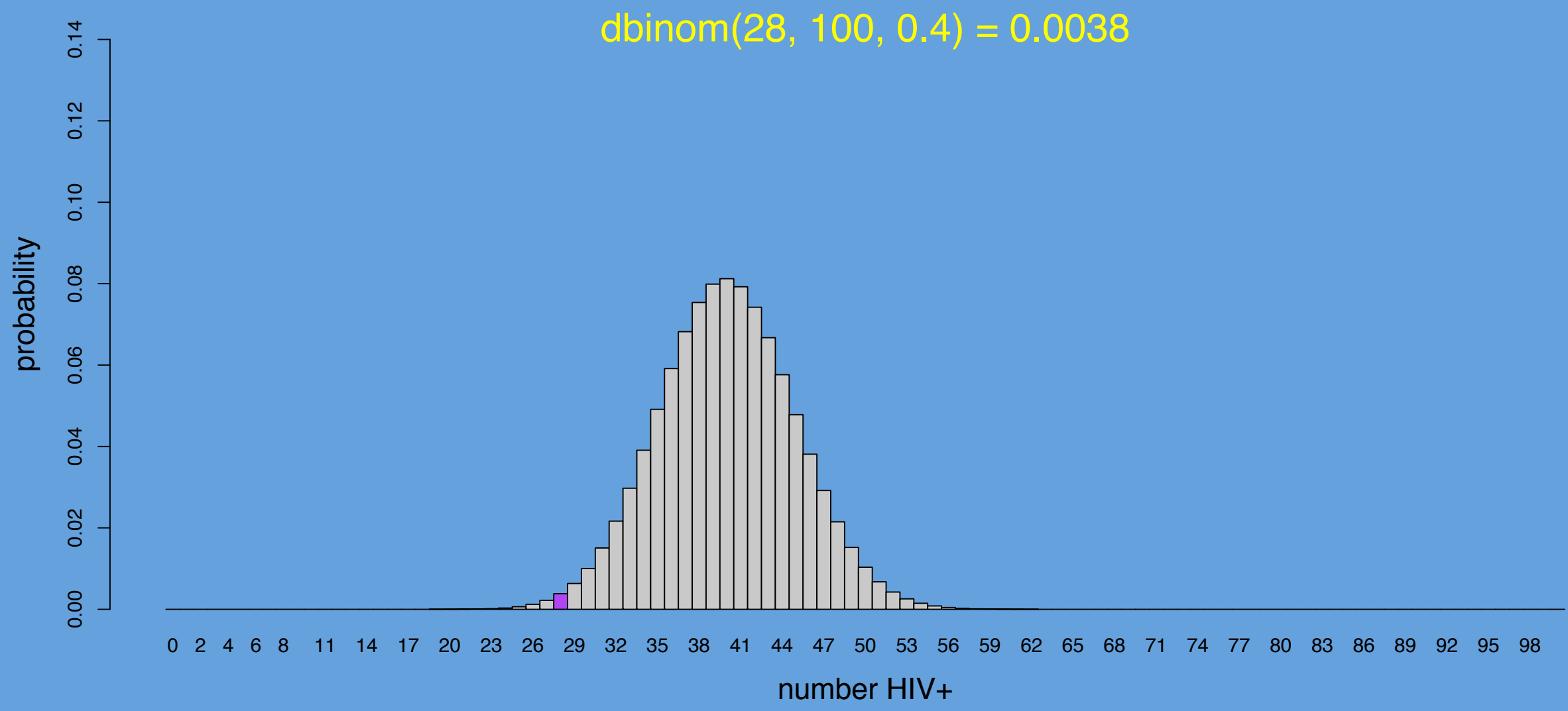




\section{Which prevalence gives the greatest probability of observing exactly $28 / 100 ?$}

hypothetical prevalence: $15 \%$

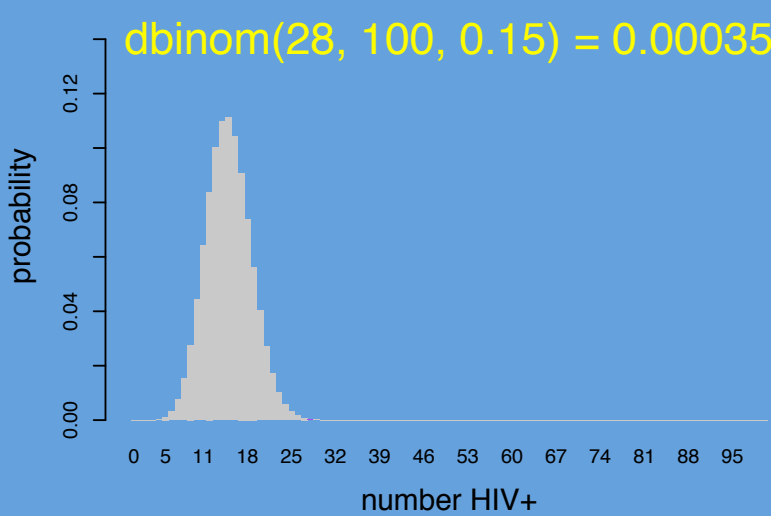

hypothetical prevalence: $30 \%$

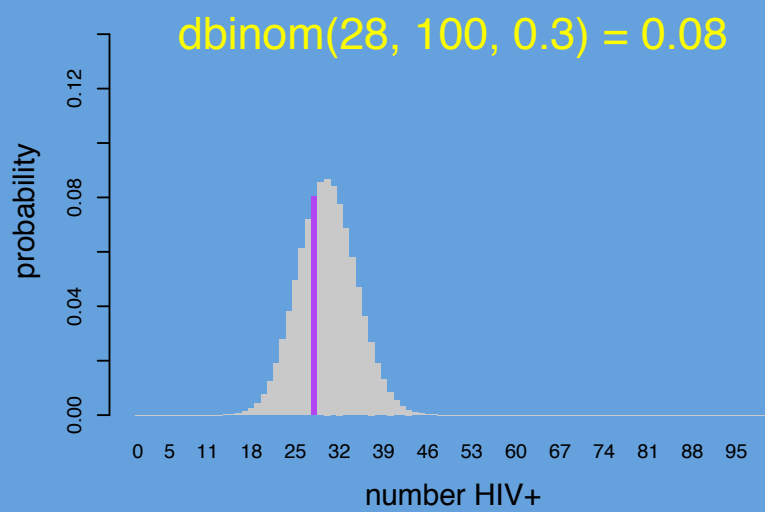

hypothetical prevalence: $20 \%$

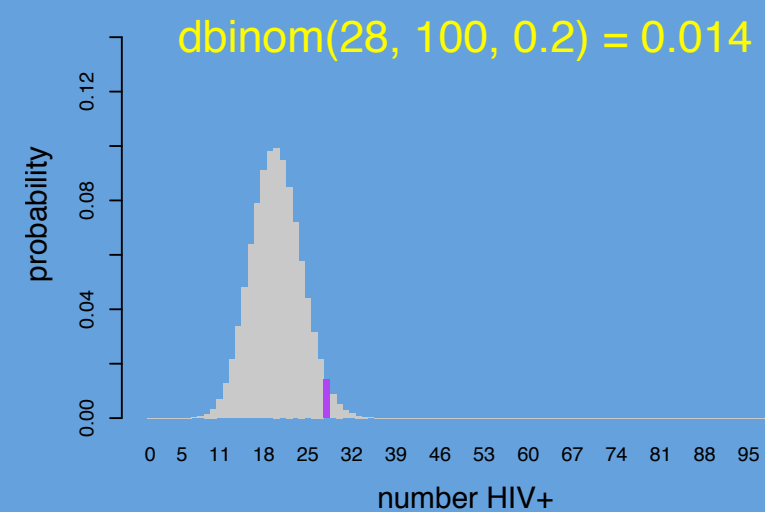

hypothetical prevalence: $35 \%$

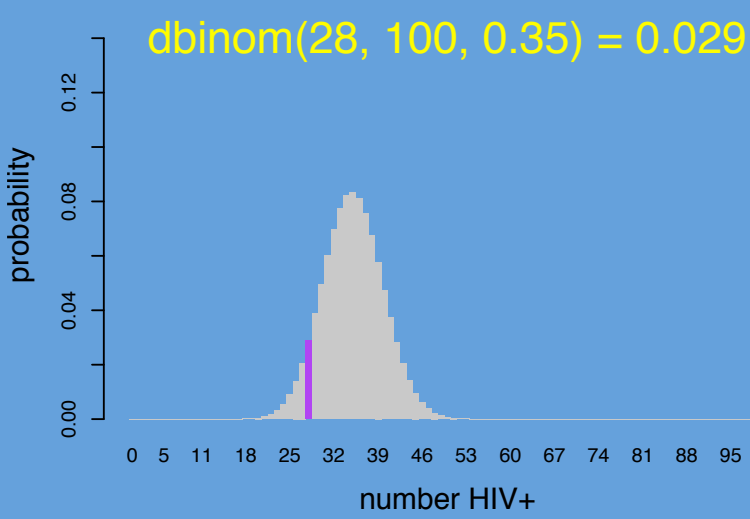

hypothetical prevalence: $25 \%$

dbinom $(28,100,0.25)=0.07$

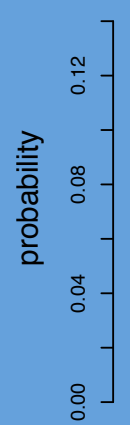

$\begin{array}{lllllllllllllll}0 & 5 & 11 & 18 & 25 & 32 & 39 & 46 & 53 & 60 & 67 & 74 & 81 & 88 & 95\end{array}$ number HIV+

hypothetical prevalence: $40 \%$

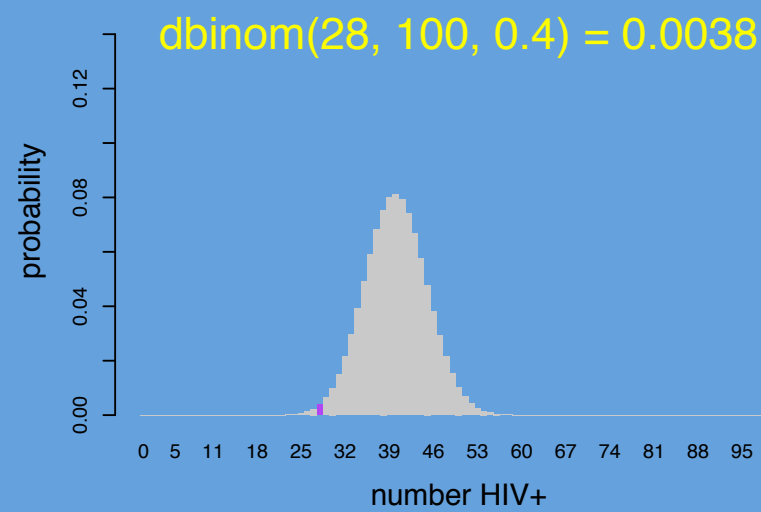




\section{Which of these prevalence values is most likely given our data?}

hypothetical prevalence: $15 \%$

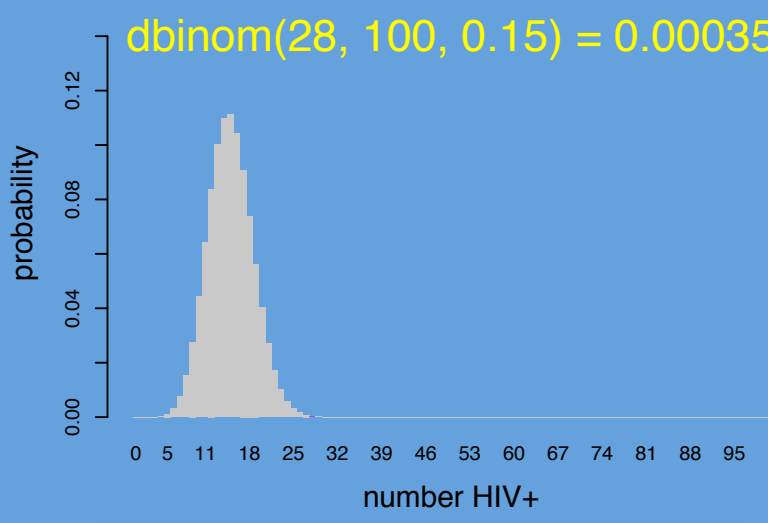

hypothetical prevalence: $30 \%$

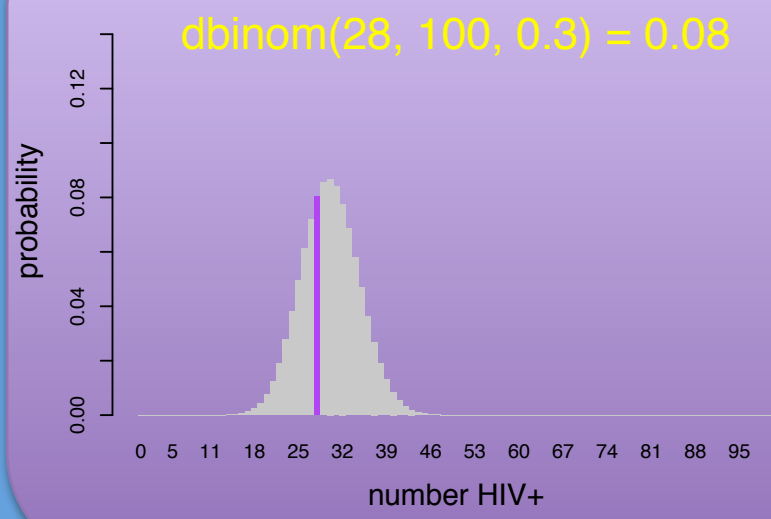

hypothetical prevalence: $20 \%$

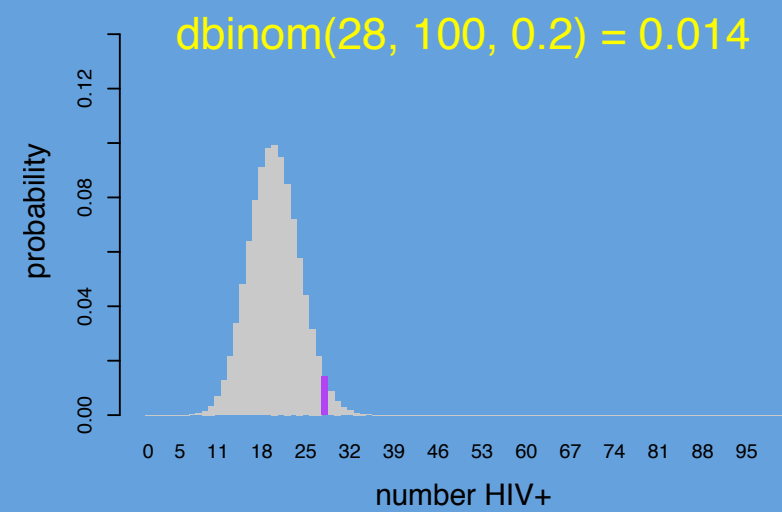

hypothetical prevalence: $35 \%$

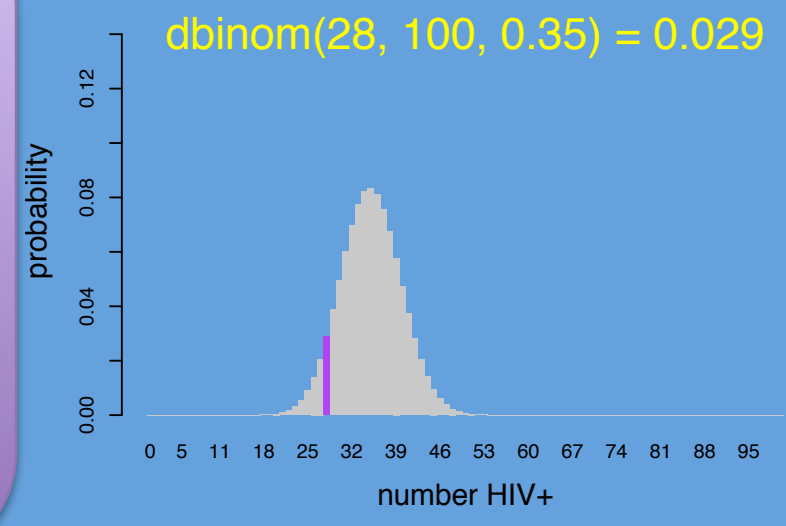

hypothetical prevalence: $25 \%$

dbinom $(28,100,0.25)=0.07$

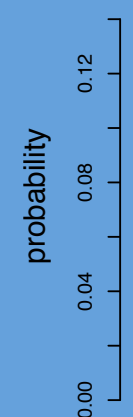

$\begin{array}{lllllllllllllll}0 & 5 & 11 & 18 & 25 & 32 & 39 & 46 & 53 & 60 & 67 & 74 & 81 & 88 & 95\end{array}$ number HIV+

hypothetical prevalence: $40 \%$

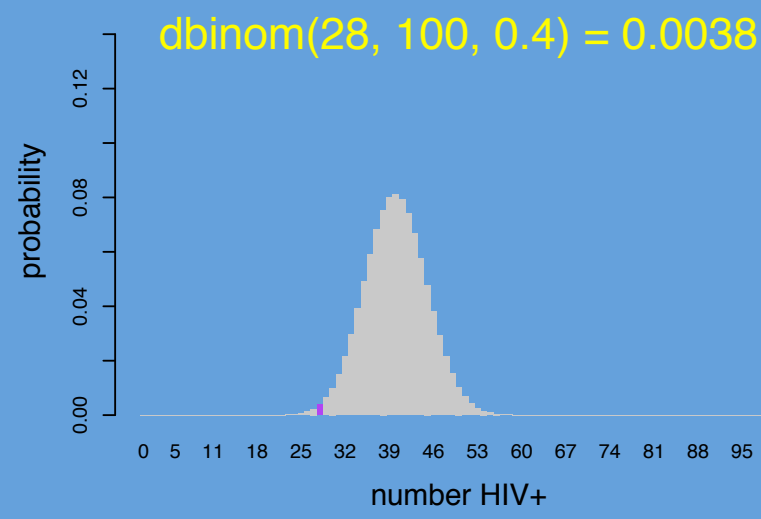




\section{$\mathrm{p}$ (our data given prevalence) $=$ LIKELIHOOD}

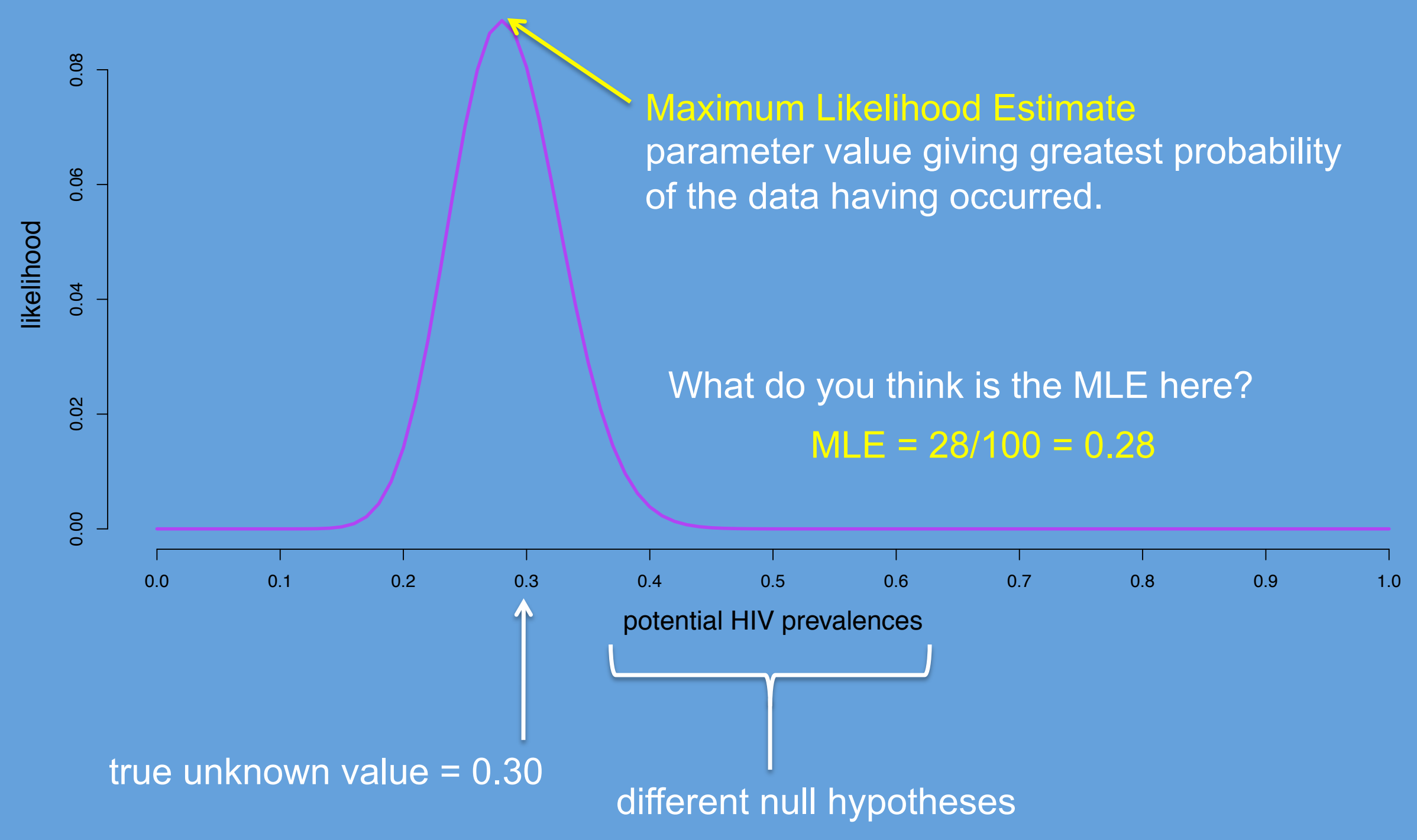




\section{Defining Likelihood}

- $L($ parameter $\mid$ data $)=p($ data $\mid$ parameter $)$

- Not a probability distribution.

$$
\text { PDF: } f(x \mid p)=\left(\begin{array}{l}
n \\
x
\end{array}\right) p^{x}(1-p)^{n-x}
$$

- Probabilities

taken from many different distributions.

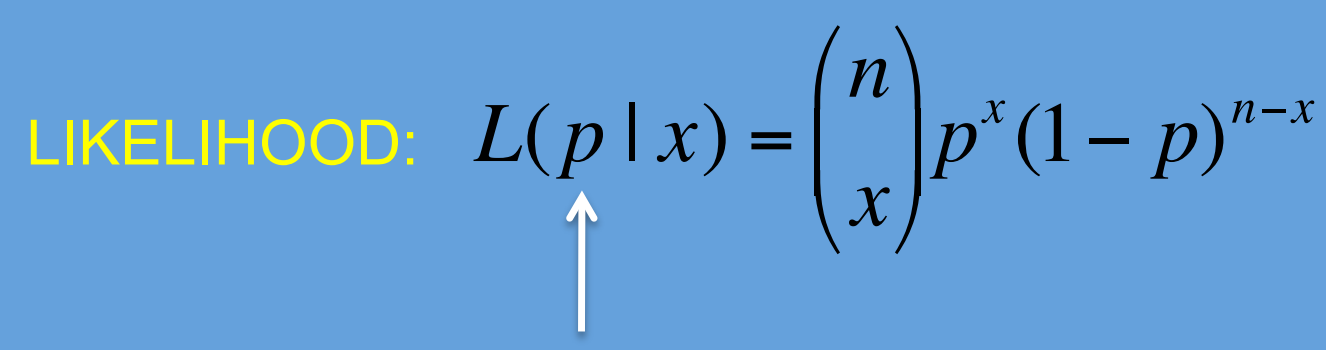

function of $p$ 


\section{Deriving the Maximum Likelihood Estimate}

maximize

$$
L(p)=\left(\begin{array}{l}
n \\
x
\end{array}\right) p^{x}(1-p)^{n-x}
$$

maximize

$$
\log \left(L(p)=\log \left[\left(\begin{array}{l}
n \\
x
\end{array}\right) p^{x}(1-p)^{n-x}\right]\right.
$$

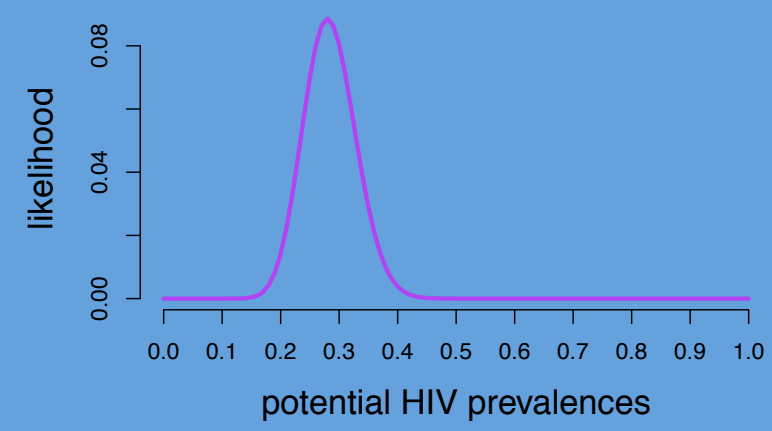

minimize

$$
l(p)=-\log \left[\left(\begin{array}{l}
n \\
x
\end{array}\right) p^{x}(1-p)^{n-x}\right]
$$
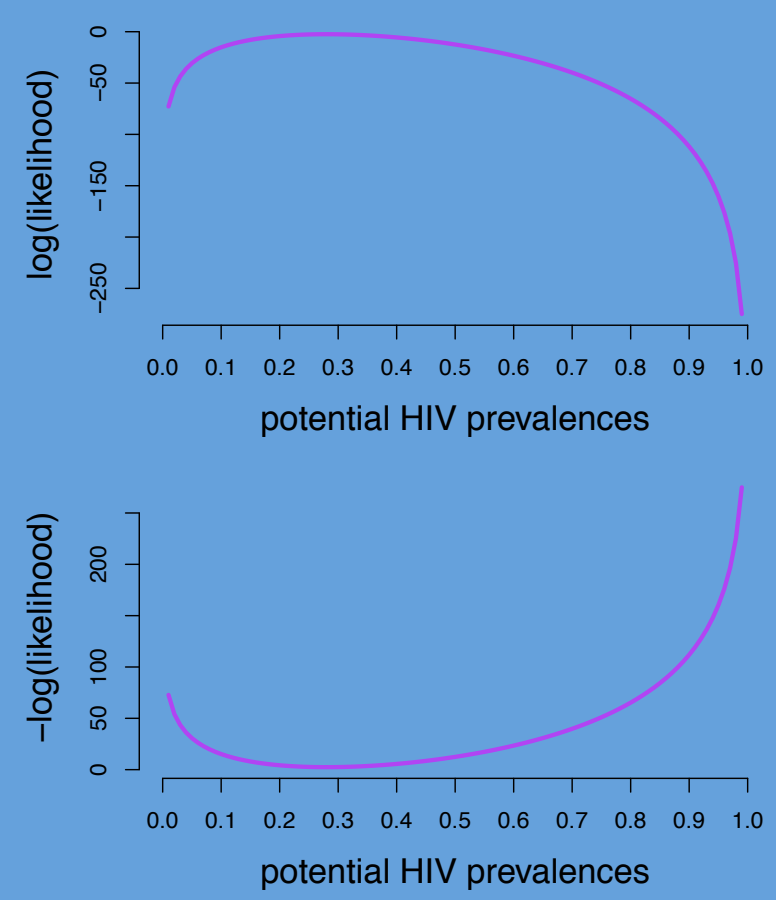


\section{Deriving the Maximum Likelihood Estimate}

$$
\begin{aligned}
& l(p)=-\log (L(p))=-\log \left[\left(\begin{array}{l}
n \\
x
\end{array}\right) p^{x}(1-p)^{n-x}\right] \\
& l(p)=-\log \left(\begin{array}{l}
n \\
x
\end{array}\right)-\log \left(p^{x}\right)-\log \left((1-p)^{n-x}\right) \\
& l(p)=-\log \left(\begin{array}{l}
n \\
x
\end{array}\right)-x \log (p)-(n-x) \log (1-p)
\end{aligned}
$$




\section{Deriving the Maximum Likelihood Estimate}

$$
\begin{array}{ll}
l(p)=-\log \left(\begin{array}{l}
n \\
x
\end{array}\right)-x \log (p)-(n-x) \log (1-p) & \\
\frac{d l(p)}{d p}=-\frac{x}{p}-\frac{-(n-x)}{1-p} & 0=-x+\hat{p} n \\
0=-\frac{x}{\hat{p}}+\frac{n-x}{1-\hat{p}} & \hat{p}=\frac{x}{n} \\
0=\frac{-x(1-\hat{p})+\hat{p}(n-x)}{\hat{p}(1-\hat{p})} & \hat{n}
\end{array}
$$

The proportion of positives! 


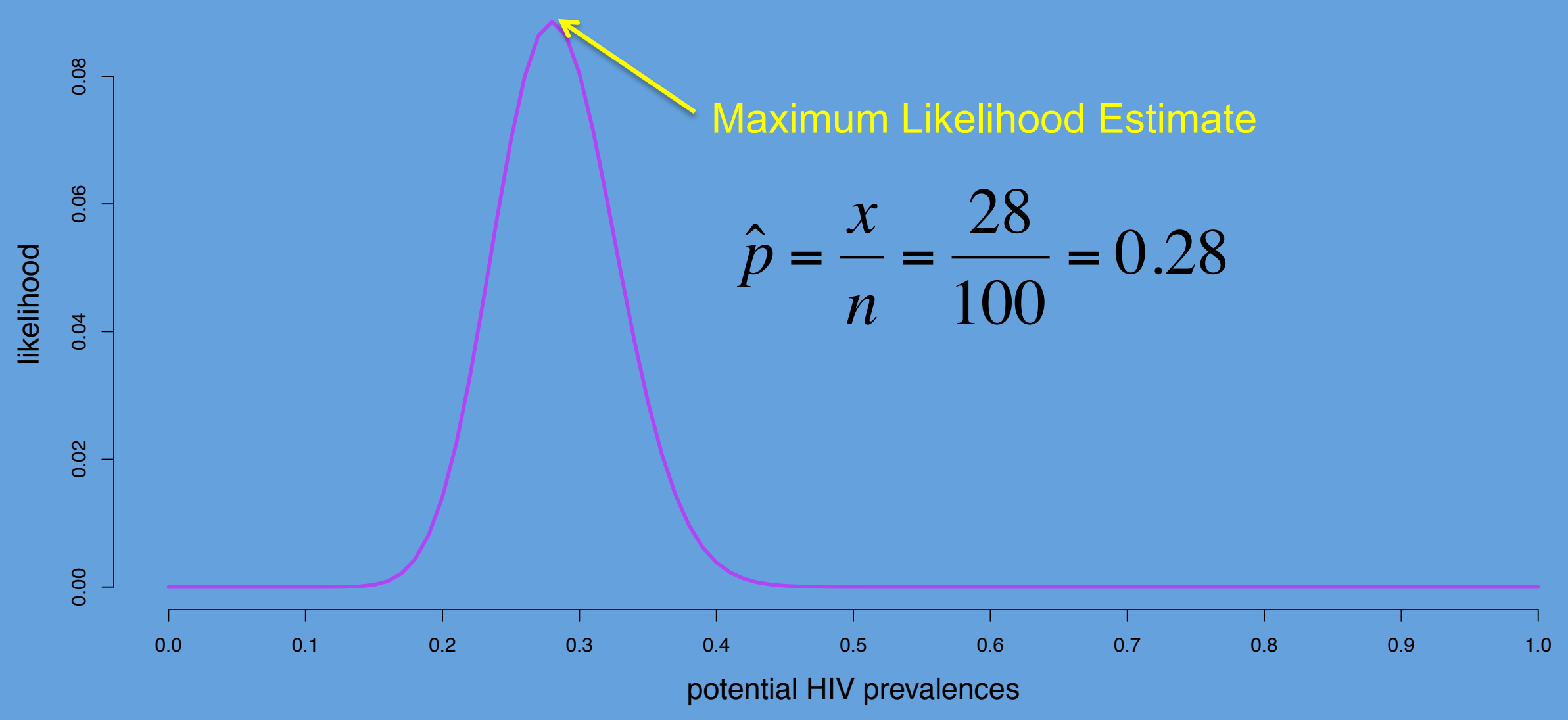




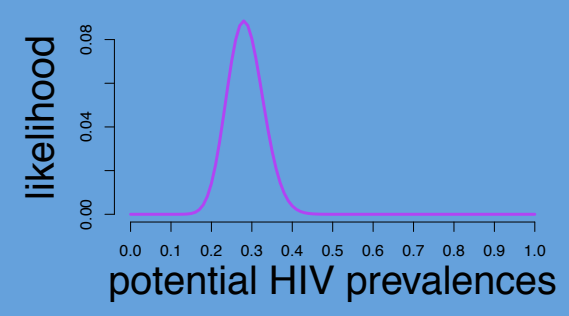

we usually minimize the -log(likelihood)

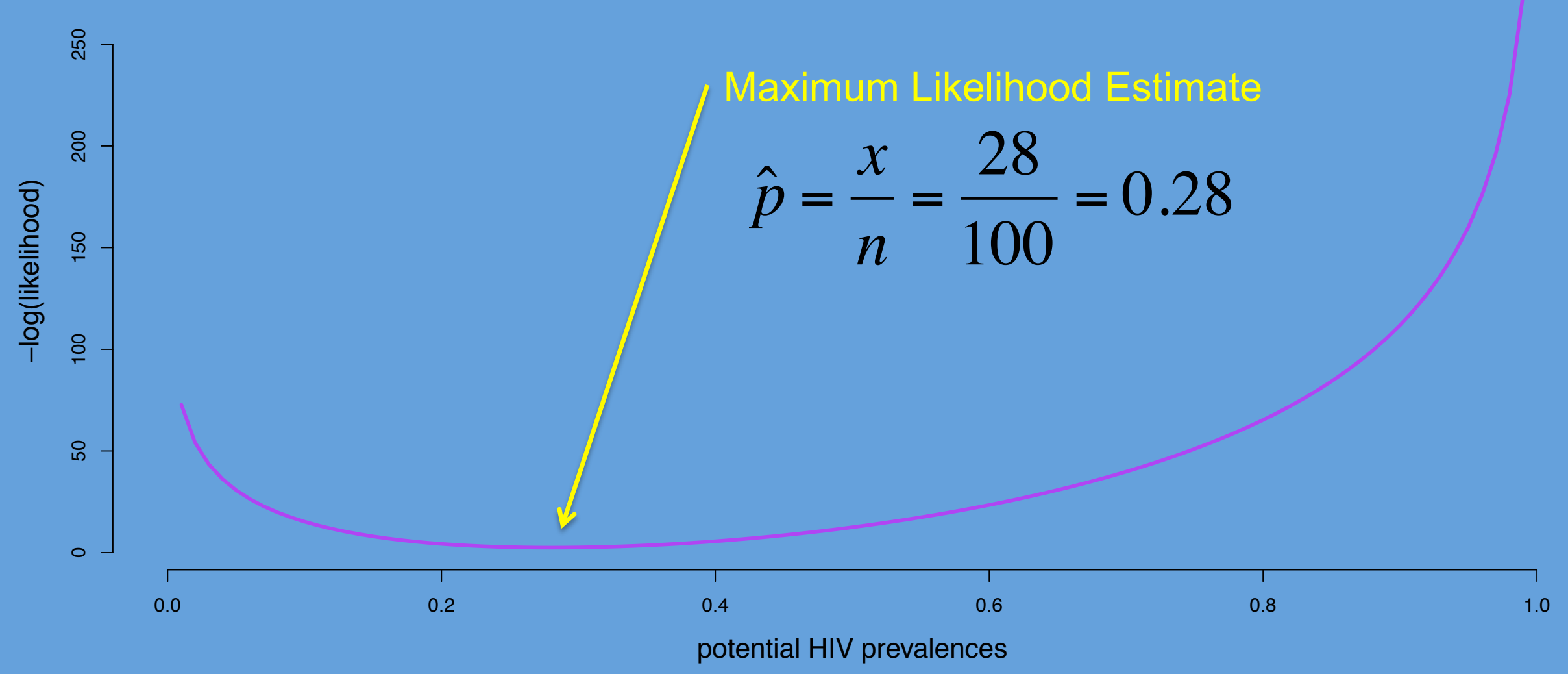




\section{Building Confidence Intervals Likelihood Ratio Test}

If the null hypothesis were true then

$$
\begin{aligned}
& -2 \log \left(\frac{L(\text { null hypoth }}{L(\text { alternative hyp }}\right. \\
& 2 l_{\text {alternative }}-2 l_{\text {null }} \sim \chi_{d f=1}^{2}
\end{aligned}
$$

PDF for $\chi_{\mathrm{dil}=1}^{2}$

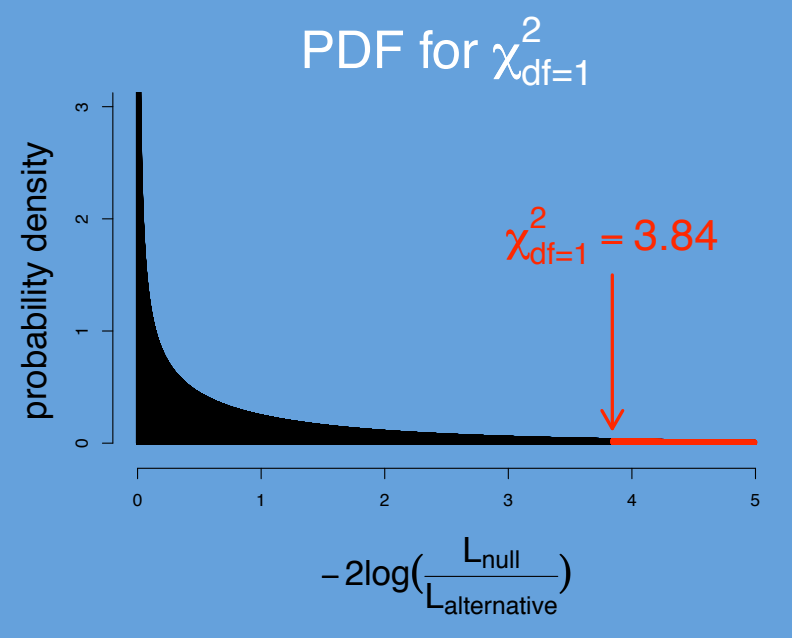

So if our $\alpha=.05$, then we reject any null hypothesis for which

$$
\begin{array}{ll}
2 l_{\text {MLE }}-2 l_{\text {null }}>\chi_{d f=1, \alpha=0.05}^{2}=3.84 & >\text { qchisq }(p=.95, \text { o } \\
2 l_{\text {MLE }}-2 l_{\text {null }}>3.84 & \text { When } I_{\text {MLE }}-I_{\text {null }}>1.941459,
\end{array}
$$$$
>\text { qchisq }(p=.95, \mathrm{df}=1)
$$$$
\text { [1] } 3.841459
$$$$
l_{\text {MLE }}-l_{\text {null }}>1.92
$$
we reject that null hypothesis. 


\section{Building Confidence Intervals Likelihood Ratio Test}

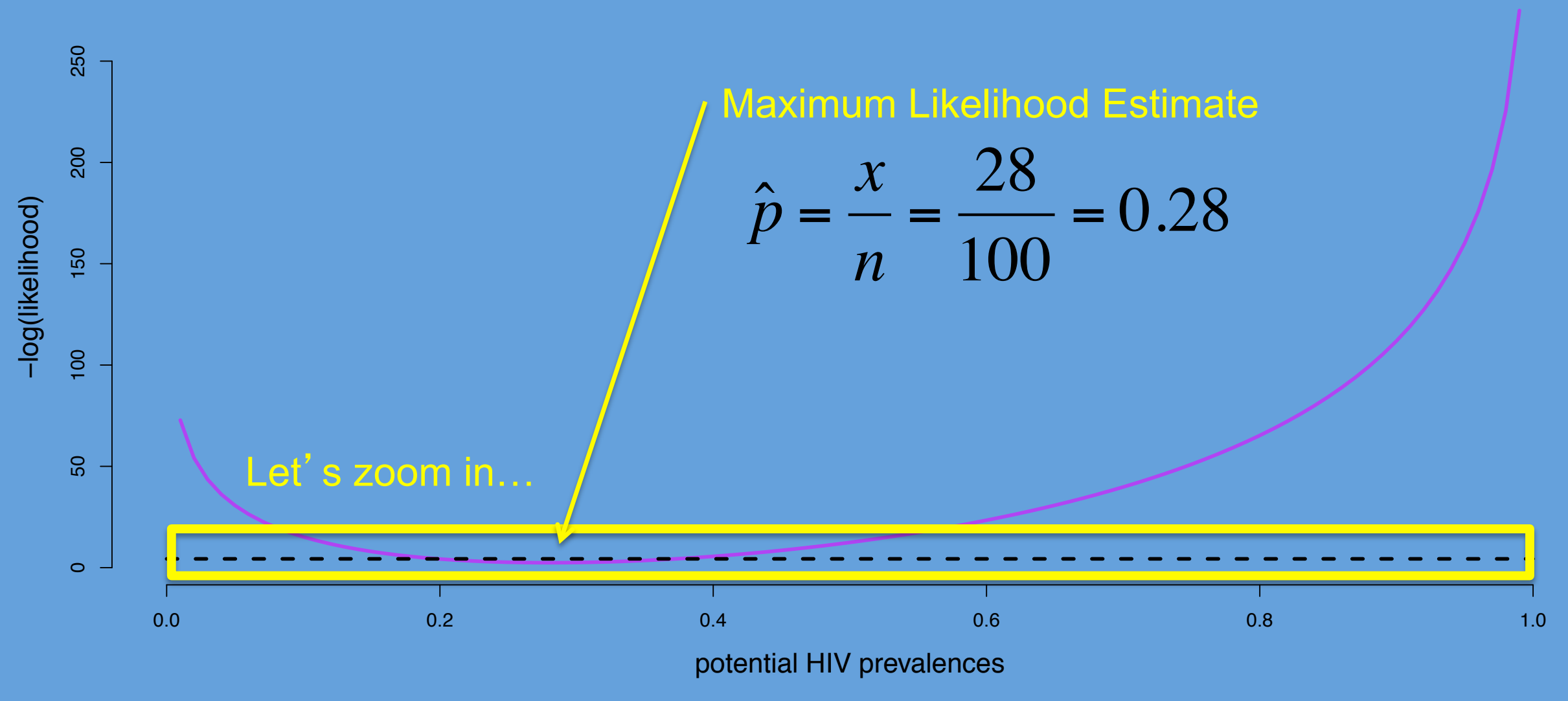




\section{Building Confidence Intervals Likelihood Ratio Test}

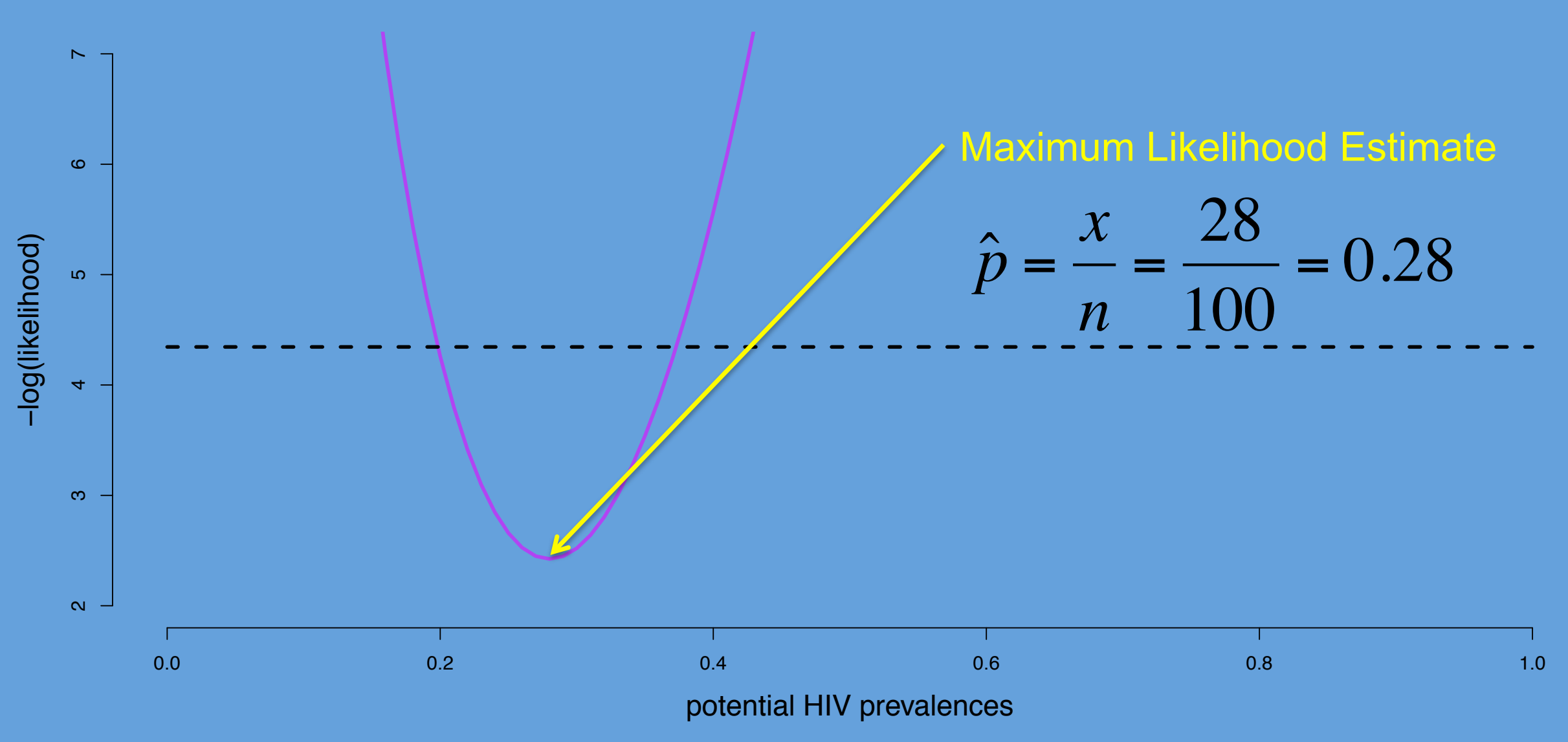




\section{Building Confidence Intervals Likelihood Ratio Test}

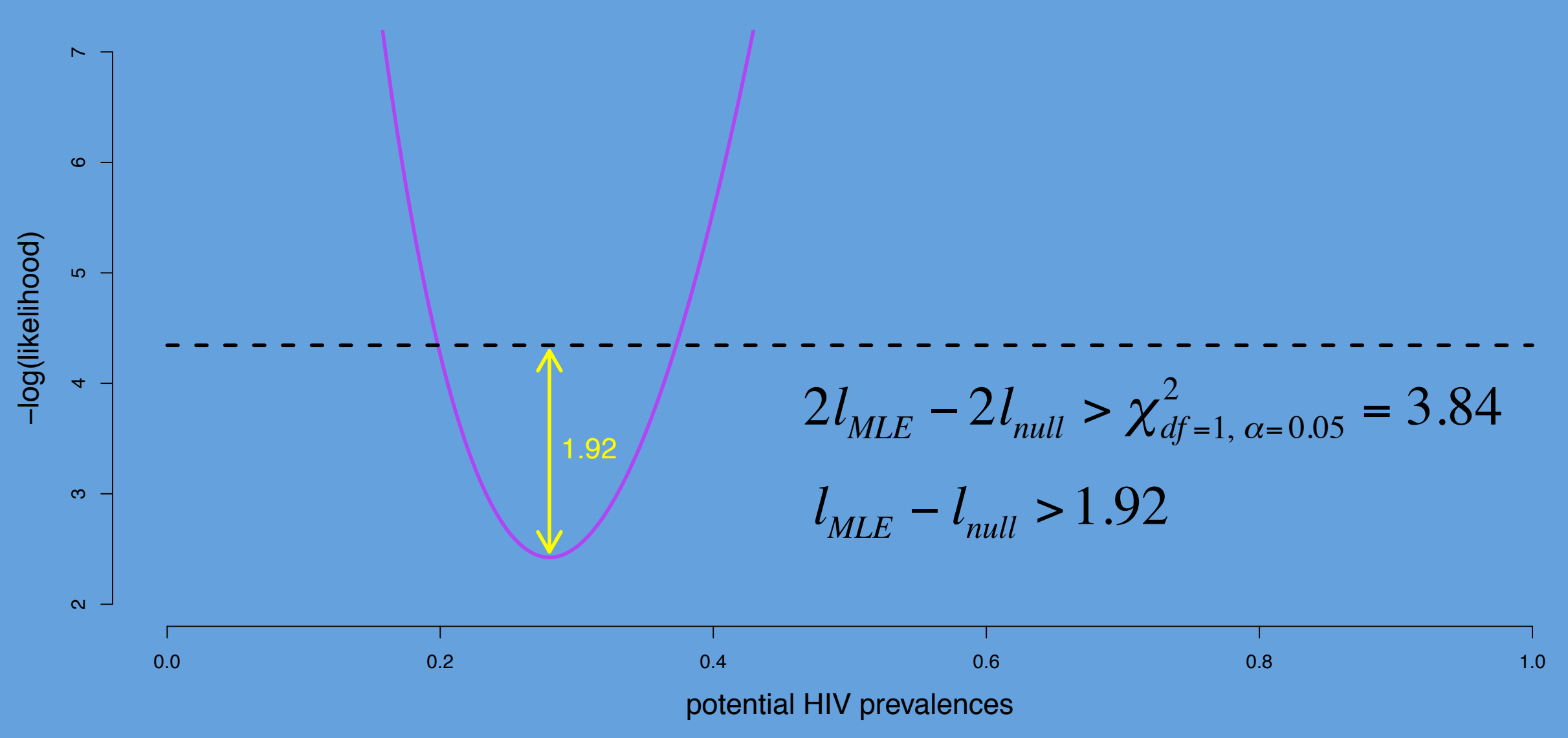




\section{Building Confidence Intervals Likelihood Ratio Test}

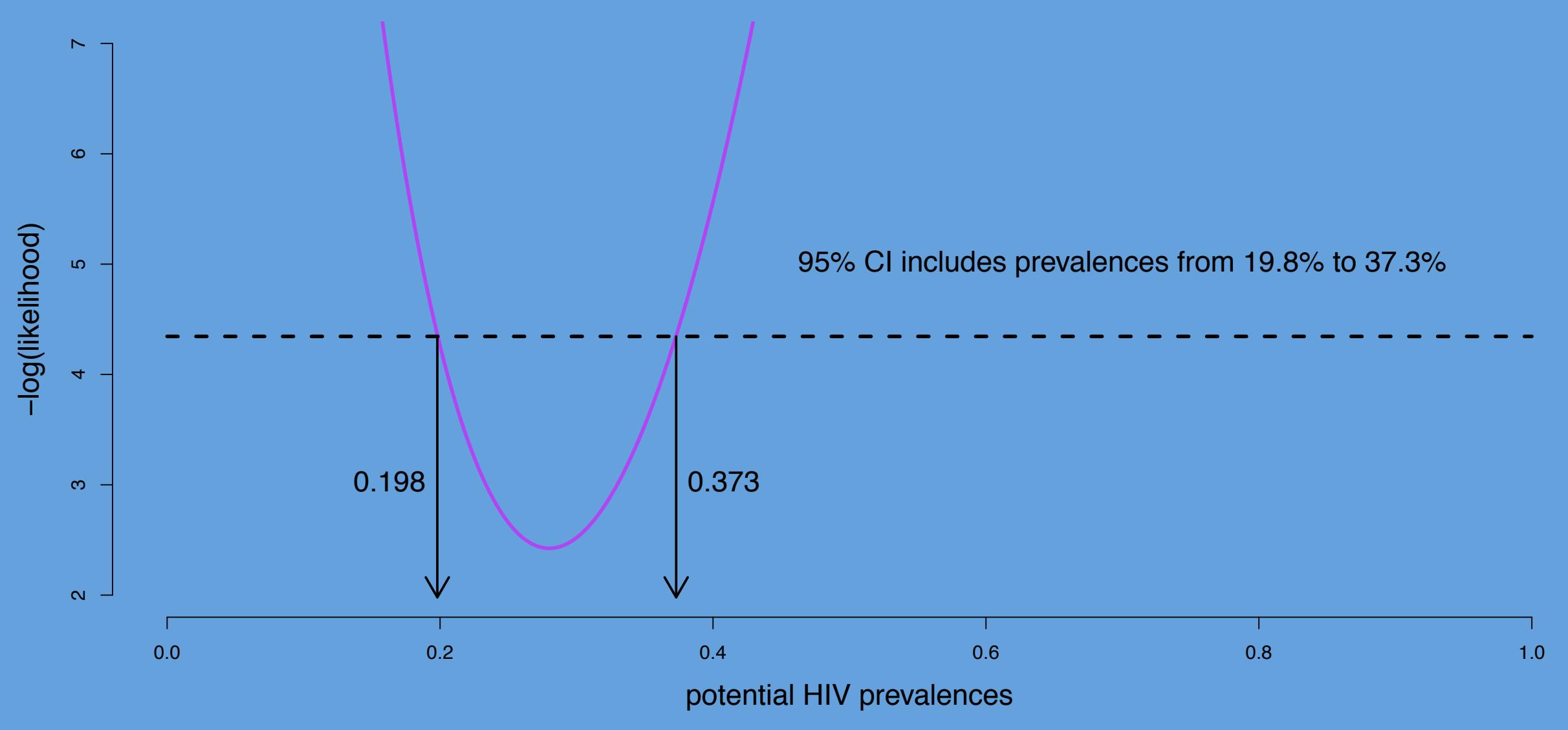




\section{Comparing Confidence Intervals}

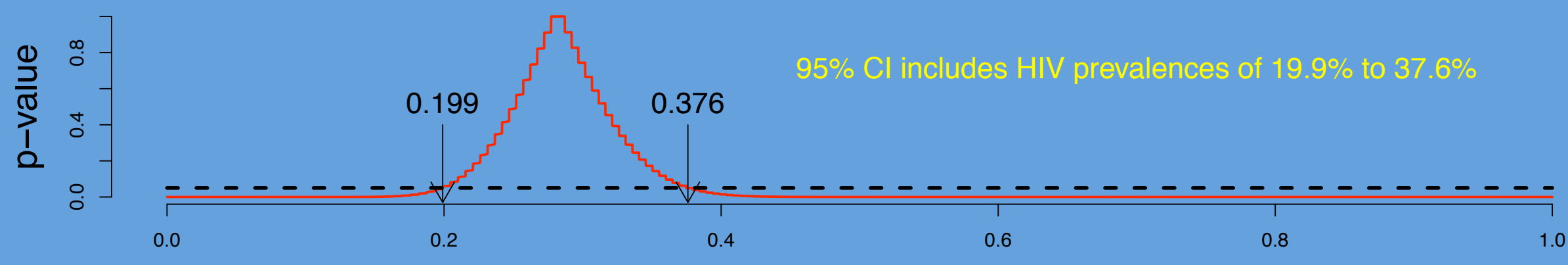

hypothetical prevalence

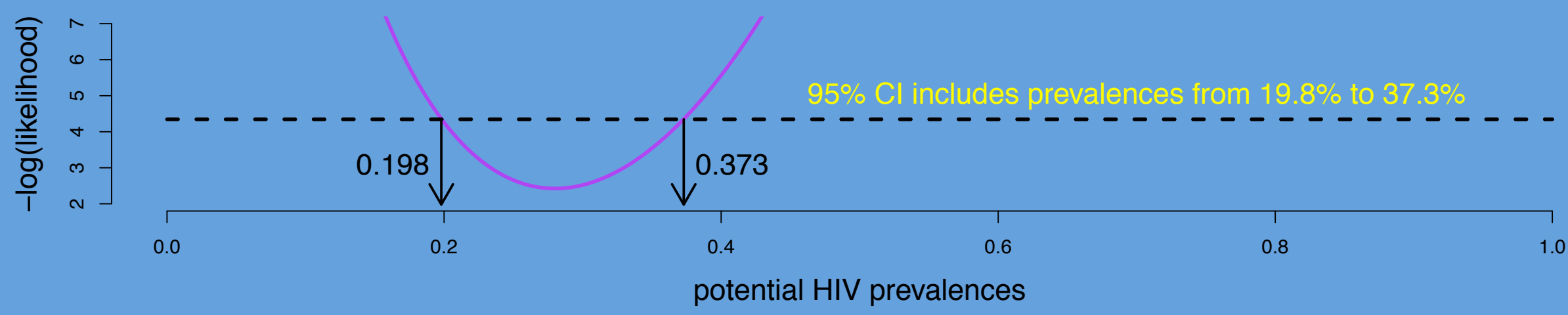




\title{
Advantages of Likelihood
}

- Practical method for

\author{
estimating parameters \\ estimating variance of our estimates
}

- Easily adaptable to different probability distributions \& dynamic models 